\title{
Interpretation of dynamic scattering from polymer solutions
}

\author{
A. Ziya Akcasu* and M. Benmouna \\ Department of Nuclear Engineering, The University of Michigan, Ann Arbor, Michigan 48109, USA
}

and Charles C. Han

Center for Materials Science, National Measurement Laboratory, National Bureau of Standards, Washington DC 20234, USA

(Received 26 October 1979)

\begin{abstract}
The theoretical results available for the interpretation of the dynamic scattering from polymer solu. tions have been re-examined. The scattering law $S(q, t)$ is formulated using the eigenfunction expansion method and the linear response theory. All previously known exact expressions of $S(q, t)$ for a single unperturbed Gaussian chain have been re-derived using the first method to demonstrate the interrelationships among the various approaches to calculation of $S(q, t)$. The results are cast into new forms which, in many cases, are more convenient for both numerical and analytical discussions. The infinite chain results are obtained from the exact closed expression of $S(q, t)$ for ring polymers as a special case as $N \rightarrow \infty$. Questions like the effect of the draining parameter on the shape of $S(q, t)$, the positive definiteness of the diffusion tensor, and the possibility of measuring the eigenvalue of the first internal mode through light scattering, have been included in the discussions.

A new method has been proposed for the interpretation of the dynamic scattering experiments in terms of the initial slope, $\Omega$, of $\ln S(q, t)$. The quantity $\Omega$ can also be identified as the first cumulant of $S(q, t)$. The advantage of this method is that $\Omega(q)$ can be calculated for all $q$ values as a function of temperature and concentration by combining the linear response theory and the blob model of chain statistics. Consequently, one is not restricted to the asymptotic small- and intermediate- $q$ regions in order to interpret the scattering experiments. The analytical and numerical results giving $\Omega(q)$ under various conditions have been presented. Using infinite chain results it is shown that $\Omega$ acts as a characteristic frequency in the sense that in both the small- and intermediate- $q$ regions, in $S(q, t)$ can be scaled to a $q$-independent shape function when time is expressed as $\Omega t$. This property facilitates the measurement of $\Omega$ from $S(q, t)$-data using a known shape function. The feasibility of the method has been demonstrated using light scattering data on polystyrene in toluene in the transition region between small- and intermediate $q$-regions.
\end{abstract}

\section{INTRODUCTION}

Quasi-elastic neutron and light scattering experiments on polymer solutions have been used increasingly in recent years to study polymer solution dynamics ${ }^{1}$. In these experiments one measures the intermediate scattering function $S(q, t)$ as a function of time $t$ for different values of the momentum transfer, $q$. The measurement of $S(q, t)$ directly by neutron scattering has been possible only very recently with the application of the spin-echo technique ${ }^{2}$.

Interpretation of such scattering experiments requires, ideally, a theory that can predict $S(q, t)$ as a function of $t$ and $q$ under actual experimental conditions, which are characterized by the temperature and concentration of the solution, and by a chain model consistent with the chemical structure of the polymer. Unfortunately, exact expression of $S(q, t)$ is available, at present, only for a single unperturbed $(\theta-$ condition) Gaussian chain without hydrodynamic interaction (Rouse model), and in the infinite chain limit, with hydrodynamic interaction and preaveraged Oseen tensor (Rouse-Zimm model). In this sense, a

0032-3861/80/080866-25\$02.00

(C) 1980 IPC Business Press complete interpretation of dynamic scattering experiments on polymer solutions is an unsolved problem.

Some progress has been possible via knowledge of the asymptotic behaviour of $S(q, t)$ for large and small times, and in certain ranges of $q$-values. It is known that $S(q, t)$ decays exponentially after large times with a decay constant, $D q^{2}$ where $D$ is the diffusion coefficient of the polymer as a whole. Hence, $D$ is determined readily by fitting an exponential function to $S(q, t)$-data for sufficiently large $t$. This procedure is workable only when $q R_{g} \ll 1$, where $R_{g}$ is the radius of gyration of the polymer. When this condition is not satisfied $S(q, t)$ decays below the noise level before the asymptotic exponential behaviour is reached. This is due to the contribution of the internal modes to the relaxation of $S(q, t)$ during the experiment. One may extend the $q$-range of the above procedure to larger $q$ values by representing $S(q, t)$ as superposition of two exponentials to take into account the effect of the most slowly decaying internal mode, as suggested by Pecora $^{3,4}$. However, one finds that the range of $q$ values and the time interval in which only the first internal mode is important are very narrow, as we will 
demonstrate below. The effect of the internal modes during the experiment becomes important all at once when' $q R_{g} \geqslant 2-3$ and thus makes the measurement of the decay constant of the first internal mode very inaccurate, if not impossible.

In the intermediate $q$-region defined by $q R_{g} \gg 1$ and $q a \ll 1$, where $a$ is the statistical segment length, the relaxation of $S(q, t)$ is determined by all the internal modes collectively. Using an unperturbed single Gaussian chain model, de Gennes ${ }^{5}$, and Dubois-Voilette and de Gennes ${ }^{6}$ showed that $S(q, t)$ can be expressed as a function of a single variable $\tau=\Omega(q) t$ in the double limit of $q a \rightarrow 0$ and $q R_{g} \rightarrow \infty$. They referred to the scaling factor, $\Omega(q)$, as 'characteristic frequency', and found that $\Omega(q) \sim q^{3}$ in the Zimm limit. This property of $S(q, t)$ has been used to interpret scattering experiments $s^{2,7}$ in the intermediate $q$-region by fitting the shape function, calculated for the unperturbed Gaussian chain, to $S(q, t)$-data, obtaining $\Omega(q)$ as function of $q$, and representing $\Omega(q)$ by a power law $q^{\alpha}$ to see whether the exponent $\alpha$ deviates from the theoretical value 3 . One difficulty with this procedure is that the conditions $q a \ll 1$ and $q R_{g} \gg 1$ are usually not strictly satisfied in an experiment. In the smalland large- $q$ ends of this interval, the molecular and segmental diffusion effects, respectively, become increasingly important. In addition, $S(q, t)$ displays, as a function of time, a crossover from Rouse-like behaviour to Zimm-like behaviour at a finite time. This cross over time is inversely proportional to $\left[\left(\xi_{0} / \eta_{0} a\right) / q a\right]^{4}$ where $\left(\xi_{0} / \eta_{0} a\right)$ is the draining parameter, $\xi_{0}$ is the friction coefficient per monomer, and $\eta_{0}$ is the viscosity of the solvent. Although the Zimm behaviour is always reached asymptotically after sufficiently long times, it may not always be attained before $S(q, t)$ decays below the noise level during an experiment. One final difficulty with the above approach is that the characteristic frequency, obtained in an experiment in which the conditions $q a \ll 1$ and $q R_{g} \geqslant 1$ are not quite satisfied, does not have to obey strictly a simple $q^{3}$ power law as predicted by the asymptotic theory.

In this paper we propose an alternative method for the interpretation of scattering experiments in terms of the initial slope $\Omega(q)$ of the normalized intermediate scattering function $\mathscr{S}(q, t) \equiv S(q, t) / S(q, 0)$, and a $q$ dependent shape function $f(\tau, q)$. The initial slope is defined by:

$$
\Omega(q) \equiv-\lim _{t \rightarrow 0} \mathrm{~d} \mathscr{P}(q, t) / \mathrm{d} t
$$

The short times involved in this definition are larger than the memory times of the solvent. We anticipate that the dynamics of the chain will be governed by a diffusion equation in which the solvent effects are averaged out.

The shape function is introduced through:

$$
\mathscr{S}(q, t) \equiv \exp [f(\Omega t, q)]
$$

Clearly, $f(0, q)=0$ and

$$
\left.\frac{\mathrm{d} f(\tau, q)}{\mathrm{d} \tau}\right|_{\tau=0}=-1
$$

The dependence of the shape function on $q$ and other parameters is more explicitly displayed as $f\left[\tau, q a, q R_{g},\left(\xi_{0} / \eta_{0} a\right)\right]$. We write it as $f(\tau, q)$ for brevity. In the small- and large- $q$ limits, $f(\tau, q)$ behaves as $f(\tau, q) \sim-\tau$, since $\mathscr{P}(q, t)$ decays exponentially in these $q$-ranges. In the mathematical limit of $q a \rightarrow 0$ and $q R_{g} \rightarrow \infty$, it becomes a function of $\tau$ only, and $\Omega$ coincides with the characteristic frequency introduced by Dubois-Voilette and de Gennes ${ }^{6}$ as we will demonstrate in this paper.

One of the merits of the present approach is that the initial slope $\Omega(q)$ is calculable for all values of $q$ as a function of temperature and concentration, in terms of the 'blob' model of chain statistics ${ }^{8,9}$. Thus, in an experiment, one is no longer restricted only to narrow asymptotic $q$-regions and dilute solutions at $\theta$ condition. The main difficulty with this method, however, is that the initial slope is hard to measure in the intermediate $q$-range. We avoid this difficulty by providing the shape function $f(\tau, q)$ as a function of $\tau$ for several values of $q$ in this as well as other $q$-regions, and for a few values of the draining parameter $\left(\xi_{0} / \eta_{0} a\right)$. By comparing these theoretical shape functions with the experimental $\ln \mathscr{S}(q, t)$ one can determine simultaneously both the initial slope $\Omega(q)$ and the statistical segment length $a$, or the radius of gyration, $R_{g}$, depending on the range of $q$-values involved. The experimental $\Omega(q)$ can thus be compared with its theoretical expression that includes concentration and temperature effects. The tacit assumption in this procedure, as well as those used earlier, is that the shape function is less sensitive to temperature and concentration effects than the initial slope, so that it can be approximated by its expression for a single Gaussian chain in $\theta$-condition.

In this paper, we present the theory for various formulations of the scattering function $\mathscr{P}(q, t)$. We then reproduce the exact expressions of $\mathscr{S}(q, t)$ for simple chain models, which are used later in the numerical calculation of the shape function. Next we calculate directly the initial slope $\Omega(q)$ for various chain models and experimental conditions. Finally we explain a procedure for the interpretation of scattering experiments using the above results, with application to real light scattering data on polystyrene in toluene.

We include a great deal of known theoretical results, but this is necessary both to put our method of interpretation of $\mathscr{S}(q, t)$ in a correct perspective, and to point out the interrelationships among these approaches, with comments on the limitations and validity of the theoretical results available for the interpretation of scattering experiments. There are novelties in the derivation and presentation of the existing and other results.

\section{THEORY}

\section{General formulation}

We consider $N$ monomers imbedded in a solution of volume $V$. The monomers are treated as material points, and their positions are denoted by $\mathbf{R}_{j}$. The set of numbers $\left\{\mathbf{R}_{1}, \ldots, \mathbf{R}_{N}\right\}$ determine a state of the solution. Due to possible constraints in the relative positions of the monomers, a state of the solution may be characterized by a reduced set of variables $\left\{\Gamma_{1}, \Gamma_{2}\right.$, ... which will be denoted collectively by a vector $\boldsymbol{\Gamma}$. 
We are interested in the calculation of the intermediate scattering function $S(q, t)$ in such a system. It is defined in terms of the two-time correlation function of the monomer density in the q-Fourier space. The monomer density is defined as:

$$
\rho(\mathbf{\Gamma})=\sum_{j} a_{j} \exp \left[i \mathbf{q} \cdot \mathbf{R}_{j}(\mathbf{\Gamma})\right]
$$

where $a_{j}$ is the scattering length of the $j$ th monomer. The $a_{j}$ will be taken to be zero if the $j$ th monomer does not participate in scattering. It will be non-zero only for the labelled monomers. The $S(q, t)$ is defined explicitly by:

$$
S(q, t)=\int \mathrm{d} \boldsymbol{\Gamma}_{0} \int \mathrm{d} \boldsymbol{\Gamma} \rho\left(\boldsymbol{\Gamma}_{0}\right) \rho^{*}(\boldsymbol{\Gamma}) \psi\left(\boldsymbol{\Gamma}_{0}, 0 ; \boldsymbol{\Gamma}, t\right)
$$

where $\psi\left(\boldsymbol{\Gamma}_{0}, 0 ; \boldsymbol{\Gamma}, t\right)$ is the joint probability of finding the monomers in the state $\boldsymbol{\Gamma}_{0}$ at $t=0$, and in $\boldsymbol{\Gamma}$ at time $t$. We assume that $\psi$ as a function of $\boldsymbol{\Gamma}$ and $t$ satisfies the following dynamical equation ${ }^{10,11}$ :

$$
\partial \psi / \partial t=\mathscr{D} \psi
$$

with the initial condition $\psi(0) \equiv \delta\left(\boldsymbol{\Gamma}_{0}-\boldsymbol{\Gamma}\right) \psi_{0}(\boldsymbol{\Gamma})$, where $\psi_{0}(\boldsymbol{\Gamma})$ is the equilibrium distribution function and satisfies $\mathscr{Q} \psi_{0}=0$. The symbol $\mathscr{D}$ in (3) denotes a linear, time-independent operator, operating on $\Gamma$. Explicit forms of $\mathscr{D}$ will be presented when specific model problems are discussed. Generalization of the dynamical equations in which $\mathscr{D}$ is time-dependent, and operates both on $t$ and $\boldsymbol{\Gamma}$, is possible, and may be required to take into account memory effects in the solvent. In this study we restrict ourselves to timeindependent dynamical operators only.

The formal solution of (3) is:

$$
\psi(t)=\exp [t \mathscr{D}] \psi(0)
$$

Substituting $\psi(t)$ in (2) and performing $\boldsymbol{\Gamma}_{0}$-integration, on finds:

$$
S(q, t)=\int \mathrm{d} \Gamma \rho^{*}(\Gamma) \exp [t \mathscr{D}]\left[\psi_{0}(\Gamma) \rho(\Gamma)\right]
$$

We define a new operator $\mathscr{L}$ through ${ }^{11,12}$ :

$$
\mathscr{D}\left(\psi_{0} A\right) \equiv-\psi_{0} \mathscr{L} A
$$

where $A(\boldsymbol{\Gamma})$ is an arbitrary dynamical variable. Then (4) can be written as

$$
S(q, t)=\int \mathrm{d} \boldsymbol{\Gamma} \psi_{0}(\boldsymbol{\Gamma}) \rho^{*}(\boldsymbol{\Gamma}) \exp [-t \mathscr{L}] \rho(\boldsymbol{\Gamma})
$$

or

$$
S(q, t) \equiv\langle\rho, \rho(t)\rangle
$$

where the time evolution of $\rho(t)$ is governed by the following equation of motion:

$$
\partial \rho / \partial t=-\mathscr{L} \rho
$$

Note that the cornered bracket $\langle A, B\rangle$ denotes the scalar product of two dynamical variables $A$ and $B$ with a weight-function, $\psi_{0}$. We assume that $\mathscr{L}$ is self-adjoint with respect to this scalar product, i.e., $\left\langle A, \mathscr{L}_{B}\right\rangle \equiv\left\langle\mathscr{L}_{A, B}\right\rangle$. We note that $\mathscr{L}=\mathscr{Q}+$ where $\mathscr{D}+$ is the adjoint of $\mathscr{Q}$ in the conventional sense, i.e. $\left(\mathscr{D}^{+} A, B\right) \equiv(A, \mathscr{Q} B)$, in which the scalar product implies integration without the weightfunction, $\psi_{0}$.

We shall present two formal approaches to the calculation of $S(q, t)$ through (6) and (7).

\section{Eigenfunction expansion}

Consider the eigenvalue problem ${ }^{13,14}$ :

$$
\mathscr{L}_{v_{n}}=w_{n} v_{n}
$$

The eigenvalues $w_{n}$ are all real because $\mathscr{L}$ is assumed to be self-adjoint. We further require $\mathscr{L}$ to be semi-positive definite, i.e. $\left\langle A, \mathscr{L}_{A}\right\rangle \geqslant 0$, so that $w_{n}$ are necessarily non-negative. The eigenfunctions $v_{n}(\boldsymbol{\Gamma})$ are orthonormalized as $\left\langle v_{n}, v_{m}\right\rangle=\delta_{n, m}$. The subscript $n$ stands for the set of all the numbers that characterize an eigenstate.

$S(q, t)$ is expanded formally as:

$$
S(q, t)=\sum_{n} e^{-w_{n} t}\left|\left\langle v_{n}, \rho\right\rangle\right|^{2}
$$

The characteristic frequency $\Omega(q)$, or the first cumulant of $S(q, t)$, defined in (1), is related to $w_{n}$ by:

$$
\Omega(q)=S^{-1}(q, 0) \sum_{n} w_{n}\left|\left\langle v_{n}, \rho\right\rangle\right|^{2}
$$

Using $w_{n}\left|\left\langle v_{n}, \rho\right\rangle\right|^{2}=\left\langle\rho, v_{n}\right\rangle w_{n}\left\langle v_{n}, \rho\right\rangle=\left\langle\rho, v_{n}\right\rangle\left\langle v_{n}, \mathscr{L}_{\rho}\right\rangle$, we obtain:

$$
\Omega(q)=\left\langle\rho, \quad \mathscr{L}_{\rho}\right\rangle /\langle\rho, \rho\rangle
$$

This result can be obtained directly from:

$$
S(q, t)=\langle\rho, \exp (-t \quad \mathscr{L}) \rho\rangle
$$

by differentiation and using the definition of $\Omega$.

The eigenfunctions $u_{n}$ of $\mathscr{D}$ are related to the eigenfunctions of $\mathscr{L}$ by ${ }^{13}$.

$$
u_{n}=\psi_{0} v_{n}
$$

This relation follows from $\mathscr{P}\left(\psi_{0} v_{n}\right)=-\psi_{0} \quad \mathscr{L} v_{n}=$ $-w_{n} \psi_{0} v_{n}$. The eigenvalues of $\mathscr{D}$ are $-w_{n}$. The eigenfunctions $u_{n}$ and $v_{n}$ form a bi-orthonormal set because $\left(u_{n}, v_{m}\right)=\left\langle v_{n}, v_{m}\right\rangle=\delta_{n, m}$.

Linear response theor $y^{10,11,15}$

By applying Zwanzig ${ }^{16}-$ Mori $^{17}$ projection operator techniques to (7) one obtaines the following equation for $S(q, t)$ :

$$
\frac{\partial S(q, t)}{\partial t}=-\Omega(q) S(q, t)+\int_{0}^{t} \mathrm{~d} u \varphi(q, u) S(q, t-u)
$$

where $\Omega(q)$ is the characteristic frequency as defined in $(11)$, and $\varphi(q, t)$ is the memory kernel defined by:

$$
\varphi(\dot{q}, t) \equiv\left\langle\mathscr{L}_{\rho, e^{-t(1-P)} \mathscr{L}_{(1-P)}} \mathscr{L}_{\rho}\right\rangle\langle\rho, \rho\rangle^{-1}
$$


Here $P$ is the projection operator defined by its action on an arbitrary dynamical variable $A$ :

$$
P A \equiv\langle A, \rho\rangle\langle\rho \rho\rangle^{-1} \rho
$$

Note that the second term in (14) accounts for deviation of the relaxation of $S(q, t)$ from a pure exponential decay that prevails for short times.

Equation (14) provides a very convenient starting point for approximate calculation of $S(q, t)$, when the eigenfunction expansion is not feasible, by applying the standard tools of the correlation analysis in simple liquids. For example, one may approximate $\varphi(q, t)$ by using continued fraction expansion ${ }^{18}$, mode-mode coupling theory ${ }^{19}$, perturbative techniques such as weak-coupling expansion and density expansion ${ }^{20}$ etc. However, the application of such techniques is not a trivial task, and has not yet been fully explored in polymer solution dynamics.

\section{SPECIFIC MODEL CALCULATIONS}

$\mathrm{S}(\mathrm{q}, \mathrm{t})$ without hydrodynamic interaction (Rouse Model)

The eigenvalues and eigenfunctions of $\mathscr{L}$ are exactly calculable for a single Gaussian chain when $\mathscr{D}$ is chosen to be the Kirkwood-Riseman ${ }^{21}$ diffusion operator without hydrodynamic interaction. Since there are no constraints, $\Gamma_{j} \equiv \mathbf{R}_{j}$. In this case, $\mathscr{L}$ has the form

$$
\mathscr{L}=-D_{m} \sum_{j=1}^{\mathrm{N}}\left[\nabla_{j}+\nabla_{j} \ln \psi_{0}\right] \cdot \nabla_{j}
$$

where

$$
\nabla_{j} \ln \psi_{0}=-\left(3 / a^{2}\right) A_{j l} \mathbf{R}_{l}
$$

Here, $A_{j l}$ are the elements of the nearest neighbour interaction matrix $\mathrm{A}$, and $D_{m}$ is the diffusion coefficient of a single monomer:

$$
D_{m}=\left(k_{B} T / \xi_{0}\right)
$$

with $k_{B} T$ denoting the temperature and $\xi_{0}$ the friction coefficient per segment.

The eigenvalue problem $\mathscr{L}_{v_{n}}=w_{n} v_{n}$ is solved ${ }^{14}$ by transforming to normal coordinates $\boldsymbol{\xi}_{k}$ through

$$
\mathbf{R}_{j}=Q_{j k} \boldsymbol{\xi}_{k}(k, j=1,2, \ldots, N)
$$

where $\underline{\underline{Q}}$ diagonalizes $\underline{\underline{A}}$

$$
\underline{\underline{Q}}^{-1} \underline{\underline{A Q}}=\underline{\underline{M}}
$$

Here $\mathrm{M}$ is diagonal with elements $\mu_{1}, \mu_{2}, \ldots, \mu_{N}$. The eigenvalue $\mu_{1}=0$, and the corresponding eigenvector $\xi_{1}$ $=(N)^{1 / 2} \mathbf{R}_{G}$ where $\mathbf{R}_{G}$ is the centre of mass of the polymer. In terms of the normal coordinates $\mathscr{L}$ reads:

$$
\mathscr{L}=-\sum_{k=1}^{N}\left[D_{m} \nabla_{k}^{2}-W \mu_{k} \xi_{k} \cdot \nabla_{k}\right]
$$

with

$$
W=\left(3 / a^{2}\right) D_{m}
$$

The eigenvalues of (22) are obtained as:

$$
W_{n}=D_{m} K^{2}+W \sum_{k=2}^{N}\left(m_{k}+n_{k}+p_{k}\right) \mu_{k}
$$

where $\mathbf{K}=\left\{K_{x}, K_{y}, K_{z}\right\}$ denote continuous indices $K_{x}$, $K_{y}, K_{z}$, and $m_{k}, n_{k}, p_{k}=0,1,2, \ldots$ We use the index $n$ to denote collectively the set of numbers $\left\{\mathbf{K}, m_{k}, n_{k}, p_{k}\right\}$.

The normalized eigenfunctions of $\mathscr{L}$ are:

$$
v_{n}\left(\xi^{N}\right)=(2 \pi)^{-3 / 2} \exp \left(i \mathbf{K} \cdot \xi_{1}\right) \prod_{k=2}^{N} v_{m_{k}}\left(x_{k}\right) v_{n_{k}}\left(y_{k}\right) v_{p_{k}}\left(z_{k}\right)
$$

with

$$
v_{m_{k}}\left(x_{k}\right)=\left(2^{m_{k}} m_{k} !\right)^{-1 / 2} H_{m_{k}}\left[\left(3 \mu_{k} / 2 a^{2}\right)^{1 / 2} x_{k}\right]
$$

In equation (26), $H_{n}(X)$ denotes the Hermite polynomial of degree $n$, and $\xi_{k}=\left(x_{k}, y_{k}, z_{k}\right)$.

Choosing the $x$-axis parallel to $q$, and separating the centre of mass coordinate, we obtain $S(q, t)$ from equation (9) as (see Appendix A):

$$
S(q, t)=\exp \left[-\frac{D_{m} q^{2} t}{N}\right] \sum_{m=0}^{\infty} \exp \left(-\eta_{m} t\right)\left|\left\langle v_{m} \rho\right\rangle\right|^{2}
$$

where

$$
\eta_{m}=W \sum_{k=2}^{N} m_{k} \mu_{k}
$$

and

$$
\begin{gathered}
\left.\left\langle v_{m}, \rho\right\rangle\right|^{2}=\sum_{j, l=1}^{N} \exp \left[-\frac{q^{2} a^{2}}{6} \sum_{k=2}^{N} \frac{\left|Q_{j k}\right|^{2}+\left|Q_{l k}\right|^{2}}{\mu_{k}}\right] \times \\
\prod_{k=2}^{N} \frac{1}{m_{k} !}\left[\frac{q^{2} a^{2}}{6 \mu_{k}}\left(Q_{j k} Q_{l k}^{*}+Q_{j k}^{*} Q_{l k}\right)\right]^{m_{k}}
\end{gathered}
$$

In equation (29) we allowed the transformation matrix $Q$ to be complex. The index $m$ in equations (27)-(29) denotes the set of integers $m_{2}, m_{3}, \ldots, m_{N}$.

It is possible to express $S(q, t)$ in a more compact form using equation (A5) in Appendix A, as:

$$
N^{-2} S(q, t)=\exp \left(-D_{m} q^{2} t / N\right) \sum_{n=0}^{\infty} P_{n}(q, t)
$$

where

$$
\begin{aligned}
P_{n}(q, t)= & \frac{1}{N^{2}} \sum_{j, l=1}^{N}\left\{\exp \left[-\frac{q^{2} a^{2}}{6} \sum_{k=2}^{N} \frac{\left|Q_{j k}\right|^{2}+\left|Q_{l k}\right|^{2}}{\mu_{k}}\right] \times\right. \\
& \left.\frac{1}{n !}\left[\frac{q^{2} a^{2}}{6} \sum_{k=2}^{N} \frac{Q_{j k} Q_{l k}^{*}+Q_{j k}^{*} Q_{l k}}{\mu_{k}} e^{-W \mu_{k} t}\right]^{n}\right\}
\end{aligned}
$$

In fact, $S(q, t)$ can be written in a closed form by performing the $n$-summation: 


$$
\begin{aligned}
S(q, t)= & \exp \left[-D_{m} q^{2} t / N\right] \sum_{j, l=1}^{N} \exp \left\{-\frac{q^{2} a^{2}}{6} \sum_{k=2}^{N} \frac{1}{\mu_{k}}\left[\left|Q_{j k}\right|^{2}+\left|Q_{l k}\right|^{2}-\right.\right. \\
& \left.\left.\left(Q_{j k} Q_{l k}^{*}+Q_{j k}^{*} Q_{l k}\right) \exp \left(-t W \mu_{k}\right)\right]\right\}
\end{aligned}
$$

Equations (31) and (32) were first obtained by Pecora ${ }^{3}$ in 1965 by solving $\psi=\mathscr{D} \psi$ directly using the method of characteristics $^{22}$. He obtained equation (31) by expanding (32). We have presented the above derivation through the eigenfunction expansion in order to demonstrate the interrelationship among these and other theoretical approaches. For example, equation (32) was also obtained by Shore and Zwanzig ${ }^{23}$ more recently by solving $\psi=\mathscr{D}, \psi$ with the method of cumulant expansion, in their study of dielectric relaxations. We will discuss equations (31) and (32) for closed and open chains separately.

\section{Closed chain}

In the case of a closed chain $\mathbf{R}_{j}=\mathbf{R}_{j+N}$, and the interaction matrix $A$ is symmetric and cyclic (or circulant). Such matrices can be diagonalized in general $^{14,23,24}$ by:

$$
Q_{j k}=\frac{1}{\sqrt{N}} \exp \left[i \frac{2 \pi}{N}(j-1)(k-1)\right]
$$

In the special case of nearest neighbour interaction, the non-zero eigenvalues of $\underline{\underline{A}}$ are known to be:

$$
\lambda_{k}=4 \sin ^{2}(\pi \mathrm{k} / N) ; \quad k=1,2, \ldots, N-1
$$

We note that $\lambda_{k}=\lambda_{N-k}$ so that the eigenvalues $\lambda_{k}$ are doubly degenerated in the case of a closed chain. Care must be exercised in approximating $\lambda_{k}$ by $4(\pi k / N)^{2}$ for large $N$, because the above property does not hold in this approximated form.

Substitution of equations (33) and (34) into equation (31) with $\lambda_{k} \equiv \mu_{k+1}$ yields:

$$
\begin{gathered}
P_{n}(\kappa, t)=\exp \left[-\kappa^{2}\left(4 / N^{2}\right) \sum_{k=1}^{N-1} \lambda_{k}^{-1}\right] \times \\
\times \frac{1}{N^{2}} \sum_{j, l=1}^{N} \frac{1}{n !}\left[\kappa^{2}\left(4 / N^{2}\right) \sum_{k=1}^{N-1} \lambda_{k}^{-1} \cos \left(2 \pi k \frac{j-l}{N}\right) e^{-W \lambda_{k} t}\right]^{n}
\end{gathered}
$$

where $\kappa=q R_{g}$ and $R_{g}^{2}=a^{2} N / 12$ for a closed chain.

In order to compare the relative importance of the various internal modes we calculated $P_{n}$ s explicitly for a few $n . P_{0}(\kappa)$ is found as

$$
P_{0}(\kappa) \cong \exp \left[-\kappa^{2} / 3\right]
$$

using the indentity (see Appendix C, equation C5)

$$
\sum_{k=1}^{N-1} \sin ^{-2}(\pi k / N)=\left(N^{2}-1\right) / 3
$$

We find without approximation, that $P_{1}(\kappa, t) \equiv 0$. In fact, we can prove more generally, using equations (33) in (29), that the modes decaying as $\exp \left[-W t \lambda_{k} m_{k}\right]$ are zero when $m_{k}$ is odd. When $m_{k}$ is even, they contribute to $S(q, t) / N^{2}$ with a coefficient

$$
\left|\left\langle v_{0, \ldots, m_{k} 0 \ldots, 0} \rho\right\rangle\right|^{2}=P_{0}(\kappa)\left[\left(m_{k} / 2\right) !\right]^{-2}\left[4 \kappa^{2} / N^{2} \lambda_{k}\right]^{m_{k}}
$$

where $k=1,2, \ldots(N-1) / 2$, assuming for simplicity in notation that $N$ is odd. In obtaining equation (38), we have made use of $\lambda_{k}=\lambda_{N-k}$ explicitly to remove the double degeneracy of the eigenvalues, so that a mode is now characterized by the set of occupation numbers $\left\{m_{1}, m_{2}, \ldots, m_{(N-1) / 2}\right\}$. The removal of the degeneracy is equivalent to redefining the expansion coefficients in equations (27) and (29) as:

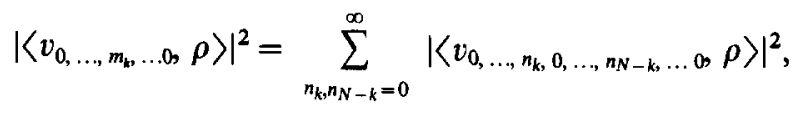

where $n_{k}+n_{N-k}=m_{k}$.

In addition to these pure modes involving a single eigenvalue, $\lambda_{k}$, there are mixed modes relaxing as exp $\left[-W\left(\lambda_{1} m_{1}+\ldots+\lambda_{(N-1) / 2} m_{(N-1) / 2}\right) t\right]$. These modes are included in $P_{n}(\kappa, t)$. One finds from (35), by first replacing the $k$-summation by $2 \Sigma_{k=1}^{N-1) / 2}$ to remove the degeneracy,

$$
P_{2}(\kappa, t)=P_{0}(\kappa) \sum_{k=1}^{(N-1) / 2}\left[\left(4 \kappa^{2} / N^{2} \lambda_{k}\right) \exp \left(-W \lambda_{k} t\right)\right]^{2}
$$

Note that there are no mixed modes in $P_{2}(\kappa, t)$. Approximating $\lambda_{k}$ as $4(\pi k / N)^{2}$ and keeping only $k=1$ mode that is dominant, we find:

$$
P_{2}(\kappa, \tau) \cong X^{2} P_{0}(\kappa) \exp [-2(\tau / X)]
$$

where $X \equiv \kappa^{2} / \pi^{2}$, and $\tau=\left(D_{m} q^{2} / N\right) t$, which expresses the time in units of $e$-folding time of the translational diffusion mode. $P_{3}(\kappa, t)$ can be shown to be:

$$
\begin{aligned}
P_{3}(\kappa, t)= & P_{0}(\kappa)\left(4 \kappa^{2} / N^{2}\right)^{3^{(N-1) / 2}} \sum_{p, q=1}\left(\lambda_{p} \lambda_{q} \lambda_{p+q}\right)^{-1} \\
& \exp \left[-W\left(\lambda_{p}+\lambda_{q}+\lambda_{p+q}\right) t\right]
\end{aligned}
$$

The dominant term is the one with $p=q=1$, which decays with a decay constant $\left(2 \lambda_{1}+\lambda_{2}\right) W$. Hence,

$$
P_{3}(\kappa, \tau) \cong P_{0}(\kappa)\left(X^{3} / 4\right) \exp [-6(\tau / X)]
$$

In summary, $S(q, t)$ is presented by explicitly displaying the first three most slowly decaying terms, as follows:

$$
\begin{aligned}
N^{-2} S(q, t) \cong & e^{-\tau} e^{-\kappa^{2} / 3}\left[1+X^{2} e^{-2 \tau / X}+\left(X^{4} / 4\right) e^{-4 \tau / X}+\right. \\
& \left.\frac{X^{3}}{4}\left(1+\frac{X^{3}}{9}\right) e^{-6 \tau / X}+\ldots\right]
\end{aligned}
$$

where we also included the dominant terms in $P_{4}$, and $P_{6}$. Note that the decay rates of $P_{6}$ and $P_{3}$ are approximately equal.

Figure 1 compares the magnitudes of the first two modes: $P_{0}(\kappa)$ and $P_{2}(\kappa, 0) \equiv P_{2}(\kappa)$, relative to the static structure factor $P(\kappa) \equiv S(q, 0) / N^{2}$. The expression for $P(\kappa)$ for a closed chain is derived later in equation (47b). The relative magnitude of the remainder, i.e. $R(\kappa)$ 


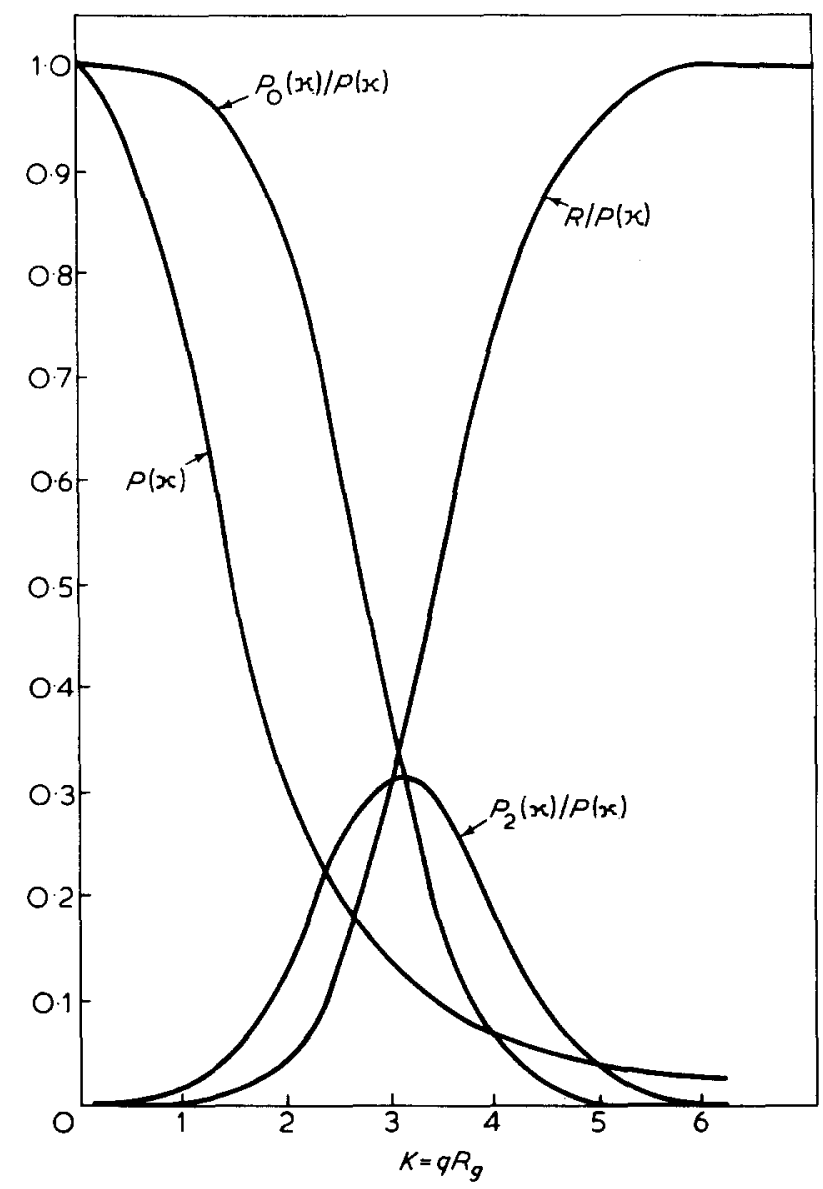

Figure 1 Comparison of the magnitudes of $P_{0}(\kappa), P_{2}(\kappa)$ and the remainder, $R(\kappa)$ as a function of $\kappa$ for a ring polymer

$\equiv P(\kappa)-\left[P_{0}(\kappa)+P_{2}(\kappa)\right]$, is also plotted. The first excited internal mode, $P_{2}(\kappa)$, decaying as $\exp \left(-2 \lambda_{1} t\right)$ becomes equal to or larger than the diffusion mode $P_{0}(\kappa)$ when $\kappa \geqslant 3$. But for such large values of $\kappa$ the remainder is larger than $1 / 3$ of the total $P(\kappa)$. The most favourable range for observing $P_{2}(\kappa)$ seems to be in the vicinity of $\kappa=2.5$ where the remainder is about $10 \%$, and $P_{2}(\kappa)$ and $P_{0}(\kappa)$ accounting respectively for about 25 and $65 \%$ of the total. In order to investigate the relative importance of the internal modes, as a function of time, we plotted $\mathscr{S}(q, t)=S(q, t) / S(q, 0)$ vs. $\tau$ $=\left(D_{m} q^{2} / N\right) t$ in Figures $2 a$ and $2 b$, for $\kappa=2.4$ and 3.2 using equation (43). The dotted curve represents the exact $\mathscr{S}(q, t)$ calculated from its closed form to be discussed next (ref equation 44). One observes in these Figures that (i) the time interval in which $P_{2}(\kappa, t)$ is above the noise level $[\mathscr{S}(q, t) \sim 0.01]$ and yet still not too small as compared with $P_{0}(\kappa, t)$ is very narrow; and (ii) the contribution of the remainder $R(\kappa, t)$ is still significant in this time interval especially in Figure $2 b$. Since we do not have any a priori knowledge of the relative magnitudes of $P_{2}, P_{0}$ and $R$ as a function of time, as well as $q$, it appears to us that the measurement of the decay constant $2 \lambda_{1}$ of the first internal mode by representing $\mathscr{S}(q, t)$ as superposition of two exponentials is at least very inaccurate, if not impossible.

The closed form of $S(q, t)$ given in equation (32) proves to be more convenient than eigenfunction expansion in studying the short time behaviour of $S(q, t)$, which is influenced by all the internal modes collectively. Substituting equations (33) and (34) into
(32) we obtain (see Appendix B):

$$
N^{-1} S(q, t)=\exp \left[-D_{m} q^{2} t / N\right]\left[e^{-\alpha \varphi_{0}(t)}+2 \sum_{s=1}^{K} e^{-\alpha \varphi_{s}(t)}\right]
$$

where

$$
\begin{gathered}
\alpha \equiv q^{2} a^{2} / 6 \\
\varphi_{s}(t)=\varphi_{s}(0)+\frac{1}{N_{k}} \sum_{=1}^{N-1}\left[\frac{1-e^{-2 \omega t[1-\cos (2 \pi k / N)]}}{1-\cos (2 \pi k / N)} \cos (2 \pi s k / N)\right] \\
\varphi_{s}(0)=N^{-1} \sum_{k=1}^{N-1} \sin ^{2}(\pi s k / N) \sin ^{-2}(\pi k / N)
\end{gathered}
$$

or (see Appendix C, equation C3):

$$
\varphi_{s}(0) \equiv|s|[1-(|s| / N)] \quad(s=0,1, \ldots \leqq N)
$$

In the derivation of equation (44) we assumed $N$ to be odd, $N=2 K+1$, for simplicity in writing. The initial slope, $\Omega$, defined by $\Omega=-\mathrm{d} \ln S(q, t) /\left.\mathrm{d} t\right|_{t=0}$ is obtained from equation (44) as:

$$
\Omega(q)=D_{m} q^{2} / N P(\kappa)
$$

where $P(\kappa) \equiv S(q, 0) / N^{2}$ is the static structure factor:

$$
P(\kappa)=N^{-1}\left[1+2 \sum_{s=1}^{K} e^{-\alpha s(1-s / N)}\right]
$$

When $N \gg 1$ so that $\alpha \ll 1$ but $\kappa^{2} \equiv \alpha N / 2$ finite, we can approximate equation (47a) by replacing the $s$ summation by an integration as:

$$
P(\kappa)=(\sqrt{2} / \kappa) e^{-\kappa^{2} / 2} \int_{0}^{\kappa / \gamma^{2}} \mathrm{~d} u e^{u^{2}}
$$

We used equation (47b) in plotting $P(\kappa)$ in Figure 1.

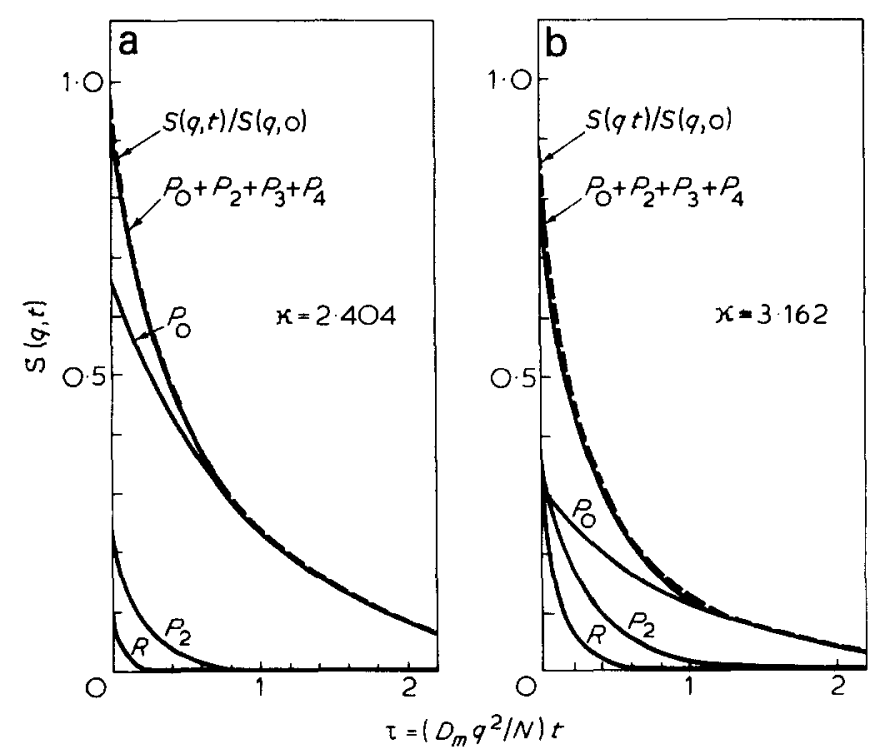

Figure 2 Decay in time of the normalized $S(q, t)$ and the various internal modes for a ring polymer and two values of $k$ 


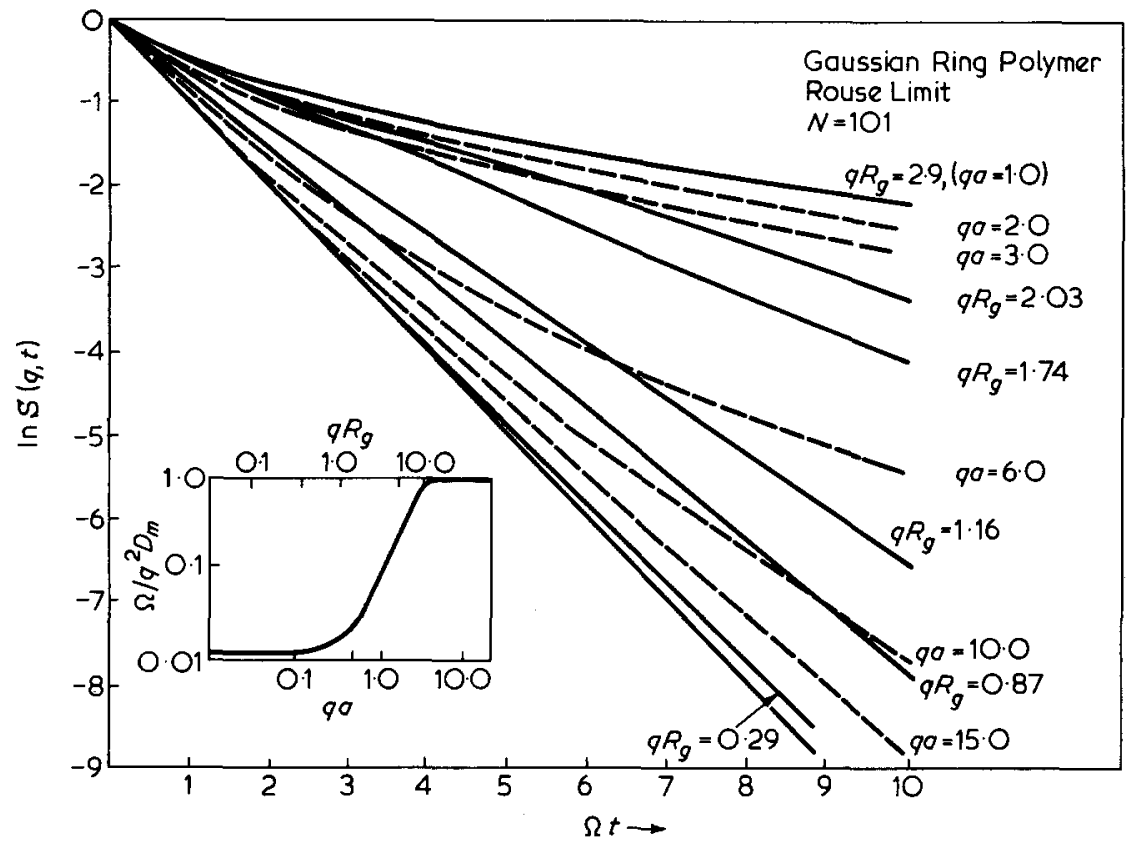

Figure 3 Variation of the shape function $\ln \mathscr{S}(q, t)$ with normalized time, $\Omega t$, for various values of $q$ in the absence of hydrodynamic interaction $(B=0)$. Also shown is the variation of the initial slope $\Omega(q)$ with (qa)

The dotted curve in Figure 2, displaying the time dependence of the normalized scattering function

$\mathscr{S}(q, t) \equiv S(q, t) / S(q, 0)$, was calculated from equations (44) and (47a) with $N=101$. Figure 3 displays the shape function $f(\Omega t, q) \equiv \ln \mathscr{S}(q, t)$ as a function of $\Omega t$ for various values of $q a$ or $\kappa=\mathrm{qR}_{g}$ where $R_{g}$ $=a(N / 12)^{1 / 2}$. The initial slopes of all these curves are equal to -1 . The shape function tends to a straight line both when $q R_{g} \leqslant 0.3$ and $q a \geqslant 15$. They cluster around the curve corresponding to $q a=1$ when $q R_{g} \geqslant 1$ but $q a \leqslant 2$. These tendencies become more apparent as $N$ increases.

Figure 3 also shows the variation of $\Omega(q)$ with $q a$. It is noted that $\Omega(q)$ tends to $\Omega(q)=D_{m} a^{2} q^{4}$ in the vicinity of $q a \sim 1$.

The infinite chain limit of $S(q, t)$ can be obtained from the following alternative expression of $S(q, t)$ (see Appendix B):

$$
N^{-1} S(q, t)=\exp \left[-\alpha \psi_{0}(t)\right]+2 \sum_{s=1}^{K} \exp \left[-\alpha \psi_{s}(t)\right]
$$

where

$$
\psi_{s}(t)=s\left(1-\frac{s}{N}\right)+\int_{0}^{2 W t} \mathrm{~d} u e^{-u} \sum_{k=-\infty}^{+\infty} I_{s+N k}(u)
$$

and $I_{n}(x)$ denotes the modified Bessel function of order $n$. Using the property $I_{n}(u) \rightarrow 0$ as $n \rightarrow \infty$ with fixed $u$, we find that only the $k=0$ term contributes when $N \rightarrow \infty$. Hence

$$
\psi_{s}(t) \cong s+\int_{0}^{2 \mu t} \mathrm{~d} u e^{-u} I_{s}(u)
$$

This approximation is valid crudely when $4 W t \ll N$.
We investigate the long chain limit of $S(q, t)$ in detail in a later section.

\section{Open chain}

For an open Gaussian chain with nearest neighbour interaction, the eigenvectors and eigenvalues of the interaction matrix $\stackrel{A}{=}$ are known to be $\mathrm{b}^{14,23,25}$ :

$$
Q_{j k}=\left(\frac{2}{N}\right)^{1 / 2} \cos \left[\frac{\pi}{N}\left(j-\frac{1}{2}\right)(k-1)\right]
$$

and

$$
\lambda_{k}=4 \sin ^{2}(\pi k / 2 N)
$$

We do not discuss the eigenfunction expansion of $S(q, t)$ in this case because it has been discussed in detail by Pecora $^{3,4}$. We only mention for completeness that $P_{0}(\kappa)$ and $P_{2}(\kappa)$ can be obtained in the present notation by substituting equations (50) and (51) into (31) as:

$$
P_{0}(\kappa)=\left(\pi / \kappa^{2}\right) \exp \left(-\kappa^{2} / 6\right)[\operatorname{erf}(\kappa / 2)]^{2}
$$

and

$$
P_{2}(\kappa, t)=4\left(\frac{\kappa}{\pi}\right)^{2} e^{-2 \kappa^{2} / 3} \sum_{p, q=1}^{N-1} \frac{1}{p^{2} q^{2}} e^{-t \bar{W} \lambda_{p}+\lambda_{p}}\left[F_{p+q}(\kappa)+F_{p-q}(\kappa)\right]^{2}
$$

where $p+q=$ even; and

$$
F_{k}(\kappa) \equiv\left(\frac{\sqrt{\pi}}{\kappa}\right) \cos \left(\frac{k \pi}{2}\right) \exp \left[\frac{\kappa^{2}}{4}-\frac{k^{2} \pi^{2}}{4 \kappa^{2}}\right] \operatorname{Re}\left\{\operatorname{erf}\left[\frac{\kappa}{2}+i \frac{k \pi}{2 \kappa}\right]\right\}
$$

In these equations, $\operatorname{erf}(x)$ denotes the conventional 
error function ${ }^{26}$. It is noted that $P_{1}(\kappa, t)$ is not zero in the case of an open chain, but it is small numerically compared with $P_{2}(\kappa)$ as discussed by Pecora ${ }^{4}$. In equations (52) $(54), \kappa=q R_{g}$ with $R_{g}^{2}=a^{2} N / 6$. In comparing the closed and open chain modes, one has to choose the radius of gyration to be the same in both cases, so that eigenvalues $\lambda_{k}$ given by equations (34) and (51) are identical for the same $k$. Since this implies $N_{\text {closed }}=2 N_{\text {open }}$, the diffusion coefficient of the closed chain will be half of that of the open chain when they have the same $R_{g}$.

The structure factor $P(\kappa)$ in the case of an open chain is ${ }^{10}$ :

$$
\begin{array}{r}
P(\kappa)=N^{-1}\left\{1+2\left(e^{\alpha}-1\right)^{-1}\left[1-N^{-1}\left(1-e^{-\alpha}\right)^{-1}(1\right.\right. \\
\left.\left.\left.-e^{-\alpha N}\right)\right]\right\}
\end{array}
$$

where $\alpha=q^{2} a^{2} / 6$. In the limit of $\alpha \ll 1$ and $N \gg 1$ with fixed $\kappa^{2}=\alpha N$, equation (55) reduces to the Debye formula:

$$
P(\kappa)=\left(2 / \kappa^{4}\right)\left(e^{-\kappa^{2}}+\kappa^{2}-1\right)
$$

which was used by Pecora ${ }^{4}$ in comparing the relative magnitudes of $P_{0}(\kappa), P_{2}(\kappa)$ and $P(\kappa)$. His conclusions with regard to the contribution of the first few internal modes in the case of open chains are essentially the same as those we obtained previously for closed chains. However, Pecora is more optimistic than us about the possibility of measuring the relaxation time of the first excited internal mode by representing the data as the superposition of two exponentials. Our conclusions are based not only on the relative magnitudes of $P_{2}(\kappa, 0) / P(\kappa)$ and $R(\kappa, 0) / P(\kappa)$ as considered by Pecora ${ }^{4}$, but also on the variation of $P_{2}(\kappa, t) / P(\kappa)$ and $R(\kappa, t) / P(\kappa)$ as a function of time.

The closed form of $S(q, t)$ for an open Gaussian chain is obtained by substituting equations (50) and (51) into (32). The result may be displayed in a compact form when $N=2 K+1$, substituting $j=K+1+r$ and $l=K+1$ $+s$ in (32) as

$$
S(q, t)=\exp \left[-D_{m} q^{2} t / N\right] \sum_{r, s=-K}^{K} \exp \left[-\alpha \varphi_{r, s}(t)\right]
$$

Following the same procedure described in Appendix $B$, we can cast $S(q, t)$ into the following form:

$$
\begin{gathered}
S(q, t)=\sum_{r, s=-K}^{\mathrm{K}} \exp \{-\alpha|r-s|- \\
\left.\alpha \int_{0}^{2 W t} \mathrm{~d} u e^{-u} \sum_{k=-\infty}^{+\infty}\left[I_{r+s+M(2 k+1)}(u)+I_{r-s+2 N k}(u)\right]\right\}
\end{gathered}
$$

In the long chain limit equation (60) reduces to:

$$
S(q, t)=\sum_{r, s=-K}^{K} \exp \left\{-\alpha|r-s|-\alpha \int_{0}^{2 W t} \mathrm{~d} u e^{-u} I_{r-s}(u)\right\}
$$

Comparing equations (61) and (48a) with (49), we find that the open and closed chain results become identical in the long chain limit, as expected.

The identity implied in equation (59) can be obtained by comparing $S(q, 0)$ calculated from equation (57) and calculated directly as done in equation (55). It is interesting to note that equation (59) also follows directly from the identity displayed in equation (45) (see Appendix C).

$S(q, t)$ with hydrodynamic interaction (Rouse-Zimm model)

Here we include the effect of hydrodynamic interactions in the formal calculation of $S(q, t)$ in terms of preaverages Oseen tensor. We refer to this model as the Rouse-Zimm model. The dynamic operator $\mathscr{L}$ in this model is ${ }^{14}$ :

$$
\mathscr{L}=-D_{m} \sum_{j=1}^{N}\left[\nabla_{j}-\frac{3}{a^{2}} A_{j l} R_{l}\right] \cdot H_{j m} \nabla_{m}
$$

where $H_{i m}$ are the elements of the Zimm matrix $\underline{\underline{H}}$ :

$$
H_{j m}=\delta_{j m}+\left(1-\delta_{j m}\right)\left(\xi_{0} / 6 \pi \eta_{0}\right)\left\langle 1 / R_{j m}\right\rangle
$$

and

$$
\left\langle\frac{1}{R_{j m}}\right\rangle=\frac{1}{a}(6 / \pi|j-m|)^{1 / 2} \text { (open chain) }
$$

where

$$
\varphi_{r, s}(t)=\varphi_{r, s}(0)+\frac{1}{N} \sum_{k=1}^{N-1} \frac{\cos [(N+r+s) \pi k / N]+\cos [(r-s) \pi k / N]}{1-\cos (\pi k / N)}\left[1-e^{-2 \omega t(1-\cos (\pi k / N)}\right]
$$

and

$$
\begin{aligned}
\varphi_{r, s}(0) & =\frac{1}{N} \sum_{k=1}^{N-1}[\cos [(N+2 r) \pi k / 2 N] \\
& -\cos [(N+2 s) \pi k / 2 N]]^{2} / 1-\cos (\pi k / N)
\end{aligned}
$$$$
\left\langle\frac{1}{R_{j m}}\right\rangle=\frac{1}{a}\left[6 / \pi|j-m|\left(1-\frac{|j-m|}{N}\right)\right]^{1 / 2} \text { (closed chain) }
$$

or (see Appendix C, equation C6)

$$
\varphi_{r, s}(0) \equiv|r-s|, \quad|r|,|s|=0,1,2, \ldots, K=(N-1) / 2
$$

In normal coordinates equation (62) is diagonalized ${ }^{27}$ as:

$$
\mathscr{L}=-\sum_{k=1}^{N} v_{k}\left[D_{m} \nabla_{k}^{2}-W \mu_{k} \xi_{k} \cdot \nabla_{k}\right]
$$


where $\mu_{k}$ are again the eigenvalues of $A$, and $v_{k}$ are the eigenvalues of $H$. Comparison of equation (65) with equation (22) shows that the effect of the hydrodynamic interaction is equivalent to multiplying the eigenvalues of each mode in the free-draining limit (Rouse model) by $v_{k}$. Hence, the new eigenvalues of $\mathscr{L}$ can be constructed as

$$
w_{n}=v_{1} D_{m} K^{2}+W \sum_{k=2}^{N} \mu_{k} v_{k}\left(m_{k}+n_{k}+p_{k}\right)
$$

The closed form of $S(q, t)$ is obtained from equation (32) as: large $N$, so that:

$$
D_{\text {ring }}=\frac{k_{B} T}{N \xi_{0}}+\frac{k_{B} T}{a \eta_{0}(N)^{1 / 2}} \frac{1}{(6 \pi)^{1 / 2}}
$$

which is a well-known result ${ }^{28}$. It is interesting to compare $D_{\text {ring }}$ with the diffusion coefficient of an open chain with the same radius of gyration, i.e. $N_{\text {ring }}$ $=2 N_{\text {open }}$. In the Zimm limit, where the Rouse term $k_{B} T / N \xi_{0}$ is neglected, we find $D_{\text {ring }} / D_{\text {open }}=0.835$, implying that a ring polymer has a larger hydrodynamic

$$
S(q, t)=\exp \left[-v_{1} D_{m} q^{2} t / N\right] \sum_{j, l=1}^{N} \exp \left\{-\alpha \sum_{k=2}^{N} \mu_{k}^{-1}\left[\left|Q_{j k}\right|^{2}+\left|Q_{l k}\right|^{2}-\left(Q_{j k} Q_{l k}^{*}+Q_{j k}^{*} Q_{l k}\right) \exp \left(-t W \mu_{k} v_{k}\right)\right]\right\}
$$

It is observed from equations (32) and (67) that the introduction of the hydrodynamic interaction corresponds to the rescaling of time for each normal mode by $v_{k}$, i.e., $t \rightarrow t v_{k}$. The eigenfunction expansion is then readily obtained from equation (27) as:

$$
S(q, t)=\exp \left[-v_{1} D_{m} q^{2} t / N\right] \sum_{m=0}^{\infty} e^{-\eta_{m} \mid}\left|\left\langle v_{m}, \rho\right\rangle\right|^{2}
$$

with

$$
\eta_{m} \equiv W \sum_{k=2}^{N} m_{k} \mu_{k} v_{k}
$$

In the case of ring polymers, the matrix $\mathrm{H}$ in equation (63) is cyclic and hence, its eigenvalues $v_{k}=$ are known ${ }^{24}$ :

$$
v_{k+1}=1+B \sum_{n=1}^{N-1}\left[n\left(1-\frac{n}{N}\right)\right]^{-1 / 2} \cos (2 \pi n k / N)
$$

where $B$ is the draining parameter:

$$
B \equiv\left(\xi_{0} / \eta_{0} a\right) / \pi \sqrt{6 \pi}
$$

Furthermore, the transformation matrix $Q_{j k}$ is still given by equation (33) which diagonalizes any cyclic matrix. Therefore, the closed form of $S(q, t)$ in the Rouse-Zimm limit is readily obtained by substituting equation (33) into (67) with $N=2 K+1$ :

$N^{-1} S(q, t)=\exp \left[-v_{1} D_{m} q^{2} t / N\right]\left[e^{-\alpha \varphi_{0}(t)}+2 \sum_{s=1}^{K} e^{-\alpha \varphi_{s}(t)}\right]$ radius due to the increased equilibrium monomer density.

The initial slope of $S(q, t)$ can be calculated from equation (71) as:

$$
\begin{gathered}
\Omega(q)=q^{2} \frac{D_{m}}{N P(\kappa)}\left\{1+2 B \sum_{s=1}^{(N-1) / 2}\left[S\left(1-\frac{s}{N}\right)\right]^{-1 / 2}\right. \\
\left.\exp \left[-\alpha s\left(1-\frac{s}{N}\right)\right]\right\}
\end{gathered}
$$

For large $N$, we replace the $s$-summation by an integral to obtain:

$$
\Omega(q)=q^{2}\left[D_{m} / N P(\kappa)\right]\left[1+\pi B(N)^{1 / 2} e^{-\kappa^{2} / 4} I_{0}\left(\kappa^{2} / 4\right)\right]
$$

where $I_{0}(x)$ is the modified Bessel function of zeroth order. $P(\kappa)$ in equations (72a) and $(72 b)$ is defined by equation (47). Burchard et al. ${ }^{29}$ calculated $\Omega(q)$ for a closed chain directly using equation (11) and without preaveraging Oseen tensor.

Figure 4 shows the variation of $\mathscr{S}(q, t)$ as a function of $\Omega(q) t$, as calculated from equations (71) and (72), for $N=101$. We observe similar trends to those obtained without hydrodynamic interaction (Figure 3). The eigenfunction expansion of $S(q, t)$ in the RouseZimm model immediately follows from equation (68) because the eigenfunctions $v_{m}$, and the expansion coefficients $\left|\left\langle v_{m}, \rho\right\rangle\right|^{2}$ are identical in the Rouse and Rouse-Zimm models for a closed chain. An explicit form similar to equations (30) and (31) can easily be obtained by replacing $t$ by $t v_{k}$ in the exponents of $P_{n}$, and $t$ by $v_{1} t$ in the first factor of equation (30).

where

$$
\varphi_{s}(t)=s\left(1-\frac{s}{N}\right)+\frac{2}{N_{k}} \sum_{k=1}^{K}\left\{\frac{1-\exp \left[-2 W t\left(1-\cos \frac{2 \pi k}{N}\right) v_{k+1}\right]}{1-\cos (2 \pi k / N)} \cos (2 \pi k s / N)\right\}
$$

We note in passing that the translational diffusion coefficient for a ring polymer is $D_{\text {ring }}=\left(D_{m} v_{1} / N\right)$ which follows from the first factor in equation (71). One can show, using equation (69), that $v_{1}=1+\pi B \sqrt{N}$ for
It is more difficult to reduce equation (67) to a simpler form in the case of an open chain because the transformation martix $Q$ which simultaneously diagonalizes $\stackrel{A}{=}$ and $\underline{\underline{\mathrm{AH}}} \underline{\underline{Q}}$, and the eigenfunctions $v_{m}$ are 


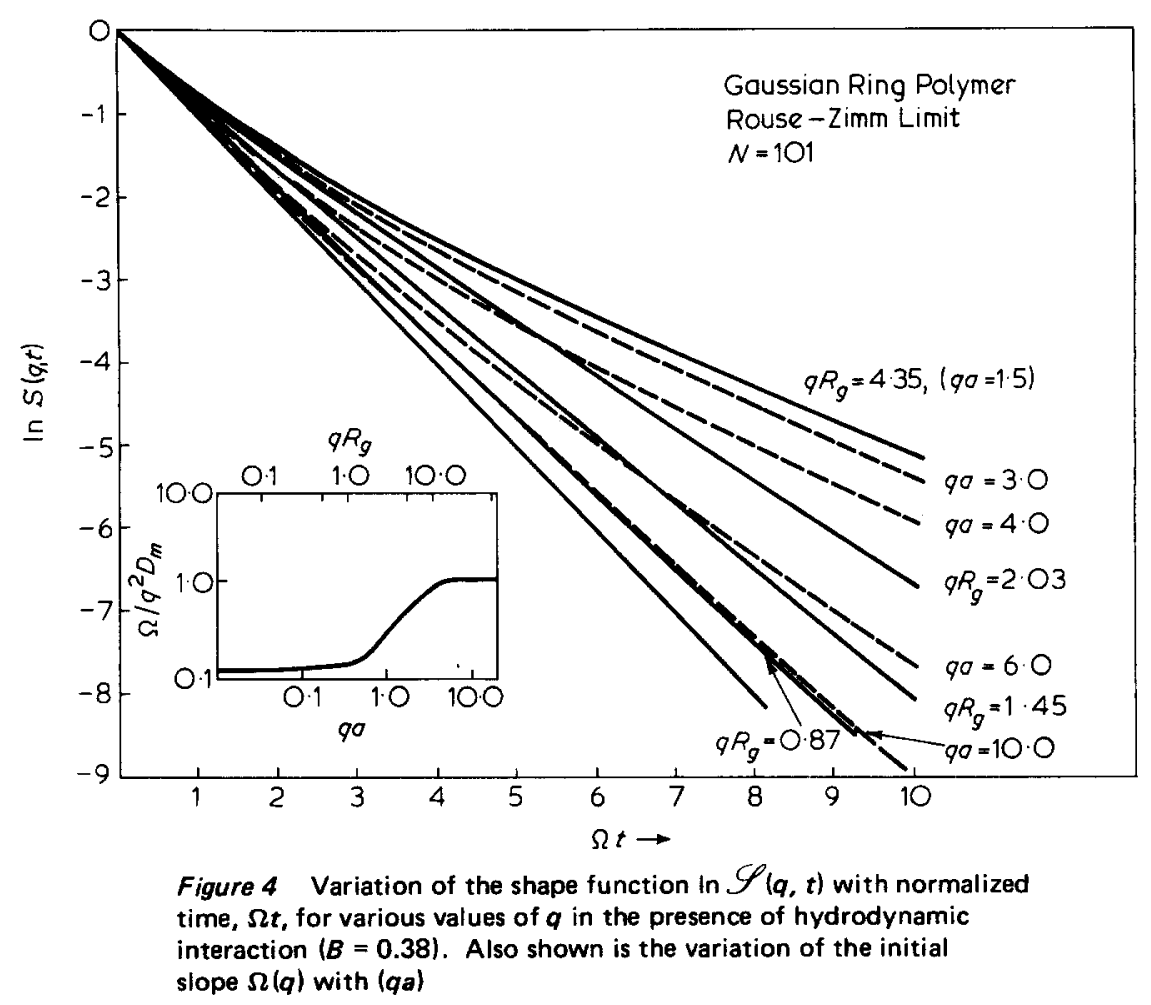

not available analytically for open chains in the Rouse-Zimm model. However, an approximate expression can still be obtained using the Rouse eigenfunctions and transformation matrix in equations (67) and (68), as suggested by $\mathrm{Zimm}^{27}$.

The analysis of $S(q, t)$ in the Rouse-Zimm limit is analytically possible in the long chain limit, for which the eigenvalues $v_{k}$ are known. Since the open and closed chains are identical in this limit we obtain $S(q, t)$ in the infinite chain limit from the closed chain result given in equations (69) and (71) with $N \rightarrow \infty$. The normalized intermediate scattering function $\mathscr{S}(q, t)$ $\equiv S(q, t) / S(q, 0)$ can be found as:

$$
\mathscr{P}(q, t)=\left[e^{-\alpha \varphi_{0}(t)}+2 \sum_{s=1}^{\infty} e^{-\alpha \varphi_{s}(t)}\right]\left[1+2 \sum_{s=1}^{\infty} e^{-\alpha s}\right]^{-1}
$$

where $\quad \varphi_{s}(t) \equiv s+\frac{1}{2 \pi} \int_{-\pi}^{\pi} \mathrm{d} p \frac{1-\exp \left(-\alpha_{p} t\right)}{1-\cos p} \cos p s$

$$
\begin{gathered}
\alpha_{p} \equiv 2 W(-\cos p)[1+2 B Z(p)] \\
Z(p) \equiv \sum_{n=1}^{\infty} \frac{\cos p n}{(n)^{1 / 2}}
\end{gathered}
$$

Equation (74) is obtained from (71b), substituting $(2 \pi / N) k=p$. In obtaining equations (75) and (76) from equation (69) we must first express the $n$-summation as $2 \sum_{n=1}^{(N-1) / 2}$, and then take the limit $N \rightarrow \infty$. The initial slope of equation (73) can be obtained either directly or as the limit of (72a) as $N \rightarrow \infty$ as:

$$
\Omega(q)=D_{m} q^{2}\left[1+2 B \sum_{s=1}^{\infty} \frac{1}{(s)^{1 / 2}} e^{-\alpha s}\right]\left[1+2 \sum_{s=1}^{\infty} e^{-\alpha s^{\top}}\right]^{-1}
$$

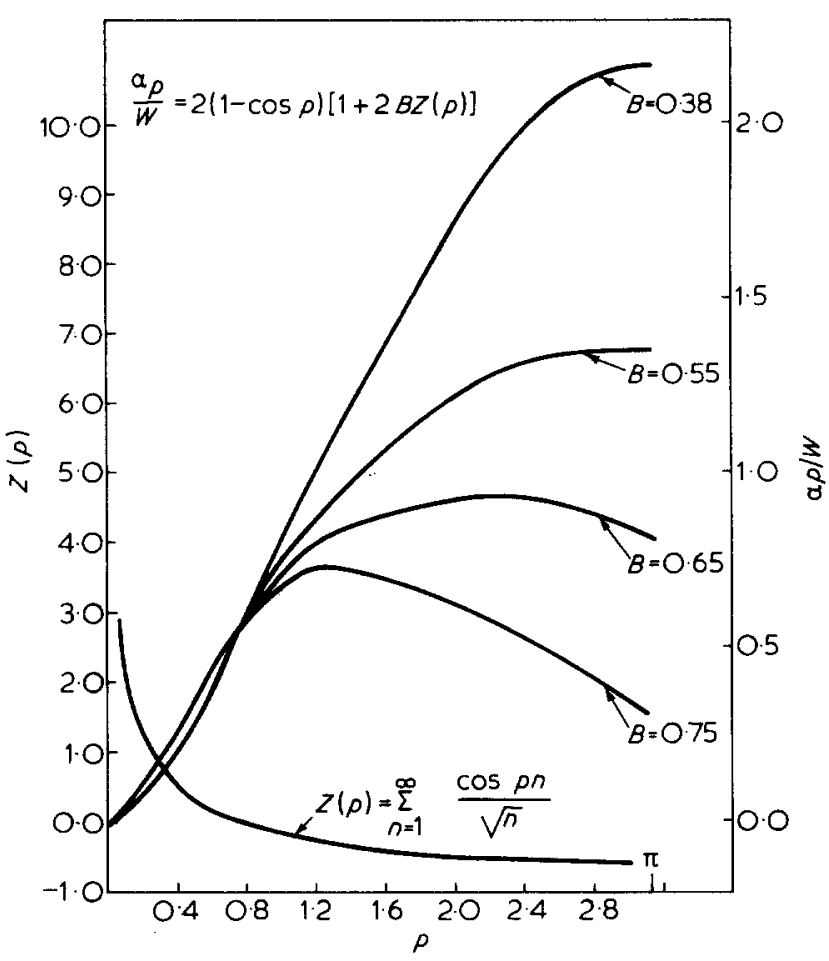

Figure 5 The variation of Zimm eigenvalues, $\alpha_{p}$, for various values of the draining parameter $B$, in the case of an infinite chain

The result in equation (73) is valid for all $q$ at all times in the case of coherent scattering from an infinite unperturbed Gaussian chain with preaveraged Oseen tensor.

A remark about the sign of $\alpha_{p}$ is in order at this point. The variation of $Z(p)$ as a function of $p$ is shown in Figure 5. For small $p, Z(p) \rightarrow(\pi / 2 p)^{1 / 2}$, and for $p=\pi$, $Z(p)=\left[(2)^{1 / 2}-1\right] \xi(1 / 2)=-0.604[\xi(x)$ is the zeta function]. We find that $\alpha_{p}$ changes sign when $B=B_{M}$ $\equiv 0.827$, which implies that the dynamical operator, $\mathscr{L}$, ceases to be positive definite when $B>B_{M}$. It is 
interesting to note that $\mathscr{L}$ is a positive definite for $B$ $=0 \cdot 38$, which corresponds to Flory's estimate ${ }^{30}$ of the draining parameter $\left(\xi_{0} / \eta_{0} a\right)$ as $5 \cdot 2$. In Figure 5, we have also plotted $\left(\alpha_{p} / W\right)$ as a function of $p$ for various values of $B$. It is observed that $\alpha_{p}$ becomes doubly degenerate when $0.827 \geqslant B \gtrsim 0.6$. This degeneracy does not occur when the Flory value of $B$ is used.

The non-positive definiteness of $\mathscr{L}$ for large values of $B$ was first pointed out by Zwanzig et al. ${ }^{31}$. They attributed it to the point particle description of the

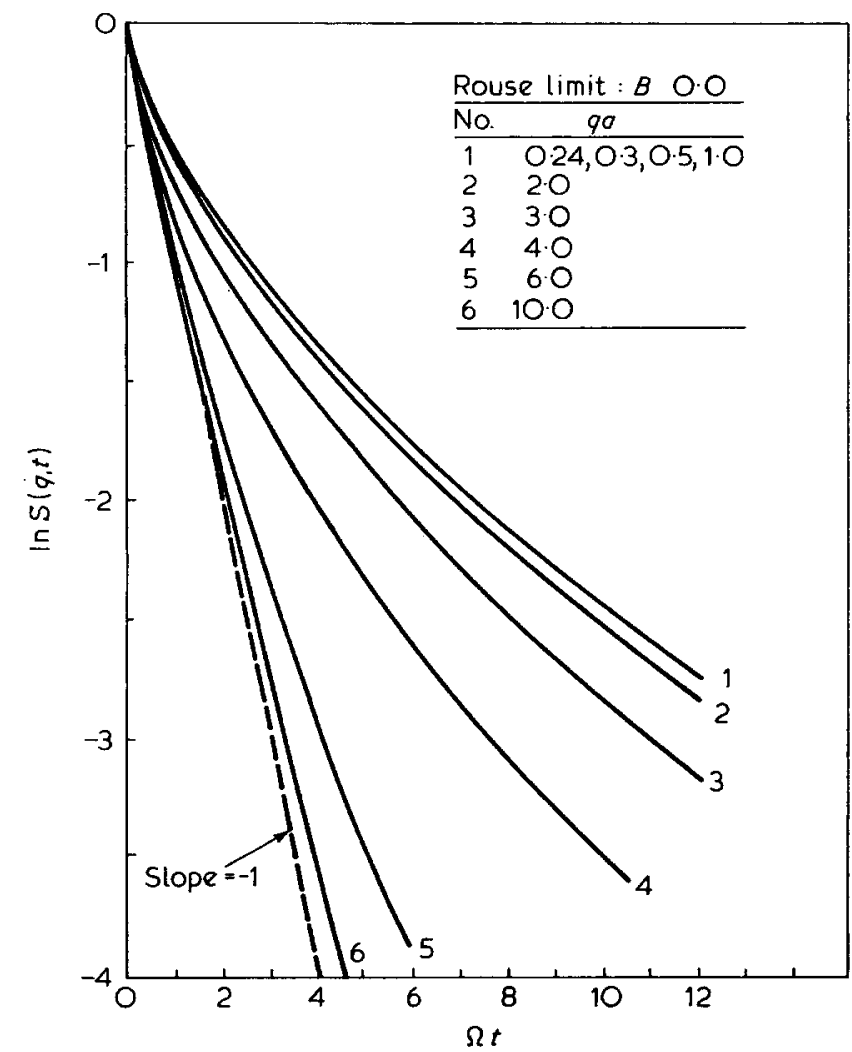

Figure 6 The variation of the shape function, in $\mathscr{S}_{(q, t)}$ with normalized time, $\Omega t$, for various values of $q a$ in the case of an infinite chain without hydrodynamic interaction monomers in the Oseen tensor approximation of hydrodynamic interactions. Ullman ${ }^{32}$ discussed ways to remedy this situation. Peterlin and Fong ${ }^{33}$ calculated $B_{M}$ for finite chains.

In Figures 6 and 7 we have plotted the variation of In $\mathscr{S}(q, t)$ as a function of $\Omega(q) t$ in Rouse, and RouseZimm models, respectively, using equations (73) and (77), for several values of $q a$. The calculated points are tabulated in Tables 1 and 2 . We observe that the curves become asymptotic to a limiting curve for all $q a$

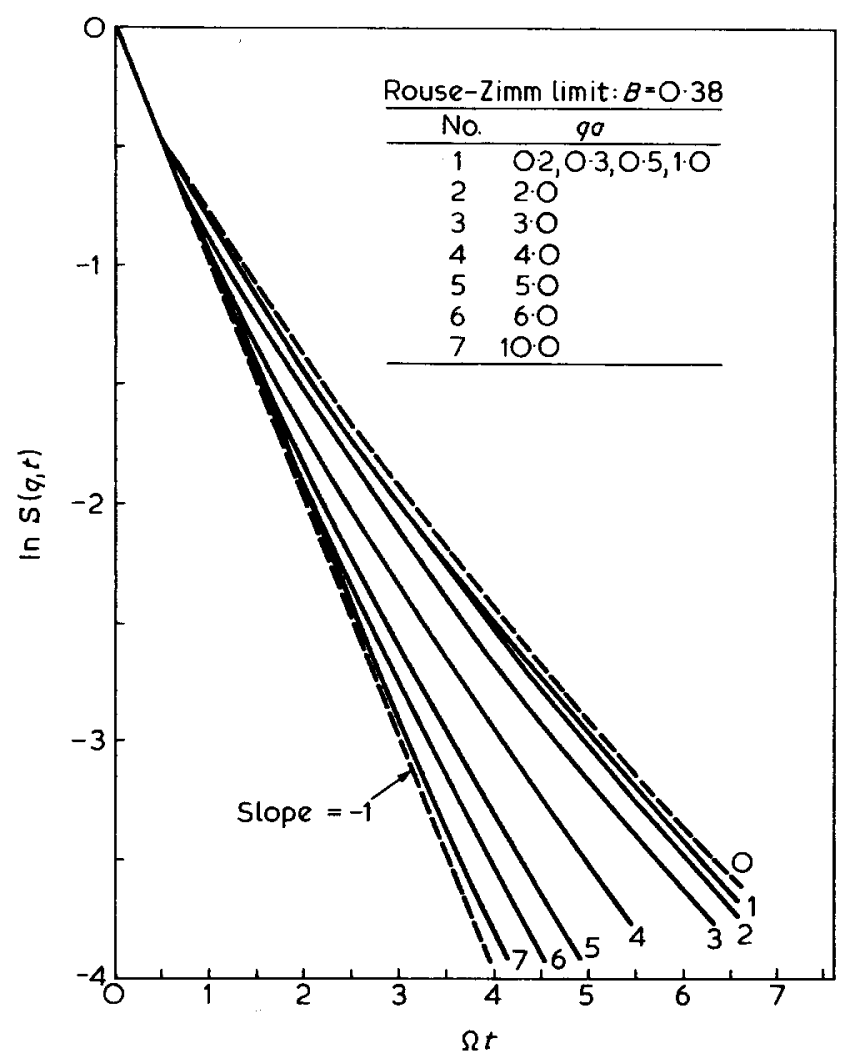

Figure 7 The variation of the shape function, $\ln \mathscr{S}(q, t)$ with normalized time, $\Omega t$, for various values of $q a$ in the case of an infinite chain with hydrodynamic in teraction

Table $1-\operatorname{Ln} \mathscr{P}(q, t)$ versus $\Omega t$ for various values of $q a$ in the long chain limit $(N \rightarrow \infty)$ and in the absence of hydrodynamic interaction (Rouse limit)

\begin{tabular}{|c|c|c|c|c|c|c|c|c|c|}
\hline$\Omega t$ & 0.24 & 0.30 & 0.50 & 1 & 2 & 3 & 4 & 6 & 10 \\
\hline No. & 1 & 2 & 3 & 4 & 5 & 6 & 7 & 8 & 9 \\
\hline 0.25 & 0.165 & 0.165 & 0.165 & 0.166 & 0.182 & 0.214 & 0.234 & 0.245 & 0.248 \\
\hline 0.50 & 0.290 & 0.290 & 0.290 & 0.291 & 0.314 & 0.38 & 0.439 & 0.475 & 0.493 \\
\hline 1.00 & 0.496 & 0.496 & 0.496 & 0.498 & 0.529 & 0.637 & 0.784 & 0.918 & 0.971 \\
\hline 1.25 & 0.587 & 0.587 & 0.587 & 0.588 & 0.623 & 0.745 & 0.931 & 0.121 & 1.245 \\
\hline 1.50 & 0.671 & 0.671 & 0.671 & 0.673 & 0.710 & 0.844 & 1.066 & 1.317 & 1.436 \\
\hline 2 & 0.826 & 0.826 & 0.826 & 0.828 & 0.870 & 1.025 & 1.306 & 1.695 & 1.887 \\
\hline 2.5 & 0.968 & 0.868 & 0.968 & 0.970 & 1.017 & 1.188 & 1.516 & 2.046 & 2.326 \\
\hline 3 & 1.100 & 1.100 & 1.099 & 1.102 & 1.152 & 1.338 & 1.705 & 2.368 & 2.753 \\
\hline 3.5 & 1.223 & 1.223 & 1.223 & 1.225 & 1.278 & 1.478 & 1.879 & 2.670 & 3.169 \\
\hline 4 & 1.340 & 1.340 & 1.339 & 1.342 & 1.398 & 1.61 & 2.041 & 2.955 & 3.573 \\
\hline 4.5 & 1.450 & 1.451 & 1.451 & 1.453 & 1.511 & 1.735 & 2.193 & 3.219 & 3.967 \\
\hline 5 & 1.557 & 1.557 & 1.556 & 1.559 & 1.620 & 1.854 & 2.337 & 3.469 & 4.351 \\
\hline 6 & 1.757 & 1.757 & 1.756 & 1.759 & 1.824 & 2.078 & 2.605 & 3.931 & 5.090 \\
\hline 7 & 1.943 & 1.944 & 1.943 & 1.945 & 2.014 & 2.286 & 2.853 & 4.349 & 5.793 \\
\hline 8 & 2.120 & 2.119 & 2.118 & 2.120 & 2.193 & 2.481 & 3.085 & 4.729 & 6.463 \\
\hline 9 & 2.285 & 2.285 & 2.285 & 2.287 & 2.363 & 2.666 & 3.303 & 5.083 & 7.106 \\
\hline 10 & 2.443 & 2.443 & 2.443 & 2.445 & 2.525 & 2.842 & 3.510 & 5.412 & 7.706 \\
\hline 11 & 2.596 & 2.596 & 2.595 & 2.597 & 2.680 & 3.011 & 3.708 & 5.721 & - \\
\hline 12 & 2.741 & 2.741 & 2.741 & 2.743 & 2.828 & 3.172 & 3.898 & - & - \\
\hline
\end{tabular}




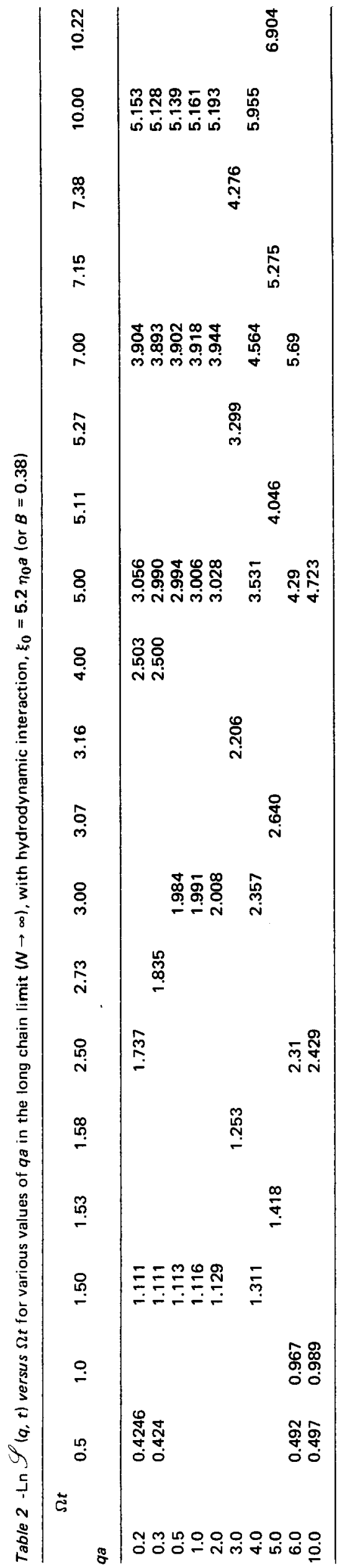

$\leqslant 1$. For $q a \geqslant 7$ they tend to a straight line with a slope $=-1$, in the time range of computations, i.e. $\Omega t \leqslant 4$. The point here is that this time range covers the time intervals relevant to an experiment because, for larger times, $\mathscr{S}(q, t)$ decays below the noise level. For example, when $\ln \mathscr{S}(q, t) \approx-4, \quad \mathscr{S}(q, t)$ is already smaller than $2 \%$. The behaviour of $\mathscr{S}(q, t)$ outside the experimental time range is of interest mainly from a theoretical point of view. We discuss the behaviour of $\mathscr{S}(q, t)$ for times $\Omega t>4$ later.

In plotting the curves in Figure 7, corresponding to the Rouse-Zimm model, we used $B=0.38$ which is the Flory value for the draining parameter. The effect of $B$ on the shape function will also be discussed later.

\section{Asymptotic behaviour of $\mathscr{S}(\mathrm{q}, \mathrm{t})$}

The form of $\mathscr{S}(q, t)$ in the intermediate $q$-region where $q a \ll 1$ and $q R_{g} \gg 1$, can be obtained easily from equation (73) in the limit $q a \rightarrow 0$. The limit $N \rightarrow \infty$ has already been taken in equation (73). Replacing $s$ and $n$-summations in equation (73) by integrations, taking $\alpha \rightarrow 0$, and considering the small-p contribution to the integral in equation (74), we obtain, in the Rouse model with $B=0$, the following expression:

$$
\mathscr{S}(q, t)=\int_{0}^{\infty} \mathrm{d} u \exp [-u-\sqrt{\Omega t} \quad g(u / \sqrt{\Omega t})]
$$

where

$$
g(u) \equiv \frac{2}{\pi} \int_{0}^{\infty} \mathrm{d} x \frac{\cos x u}{x^{2}}\left[1-\exp \left(-x^{2}\right)\right]
$$

The initial slope in this limit follows from equation (77) as:

$$
\Omega(q) \equiv \frac{1}{12}\left(k_{B} T / \xi_{0}\right) q^{4} a^{2}
$$

This result was first obtained by de Gennes ${ }^{5}$ in 1967 . The following observations are important for our later discussions:

(i) $\mathscr{S}(q, t)$ is expressed in the intermediate $q$-region as a function of a single variable, $\Omega(q) t$, which combines both $q$ and $t$. When time is scaled as $\tau=\Omega(q) t$, a universal curve as a function of $\tau$ is obtained for all $q$ values in this $q$ region. This conclusion justifies the interpretation of $\Omega(q)$ as a characteristic frequency in the sense of dynamic scaling.

(ii) $\Omega(q)$ is the initial slope of $\mathscr{S}(q, t)$ at $t=0$.

(iii) The $q$ dependence of $\Omega(q)$ follows a power law in the intermediate $q$-region (see equation 80 ).

(iv) For large times:

$$
\mathscr{S}(q, t) \rightarrow \exp \left[-(2 / \sqrt{\pi})(\Omega t)^{1 / 2}\right]
$$

where we have used $g(0)=2 / \sqrt{\pi}$

By replacing the $n$-summation in equation (76) by integration, and considering the small-p limit of $Z(p)$, we obtain in the Rouse-Zimm model $(B \neq 0)$ :

$$
\mathscr{S}(q, t)=\int_{0}^{\infty} \mathrm{d} u \exp \left\{-u-(\Omega t)^{2 / 3} h\left[u(\Omega t)^{-2 / 3}\right]\right\}
$$




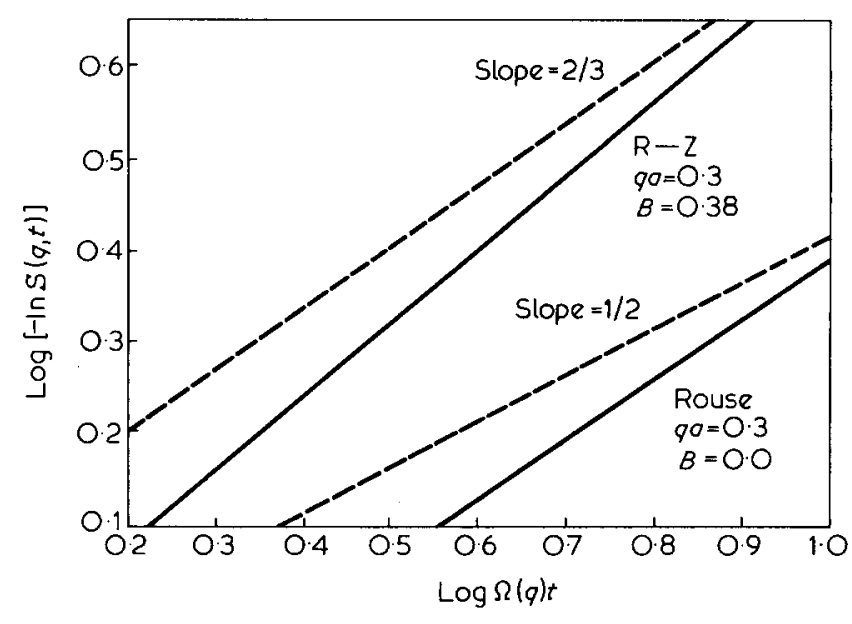

Figure 8 Comparison of the behaviour of the shape function, In $\mathscr{S}(q, t)$ at finite $\Omega t$ with its asymptotic power law behaviour at large times

where

$$
h(u) \equiv \frac{2}{\pi} \int_{0}^{\infty} \mathrm{d} x \frac{\cos x u}{x^{2}}\left[1-\exp \left(-x^{3 / 2} / \sqrt{2}\right)\right]
$$

The initial slope follows either from equation (77) or (72b) as:

$$
\Omega(q)=\frac{1}{6 \pi}\left(k_{B} T / \eta_{0}\right) q^{3}
$$

These results were first obtained by Dubois-Violette and de Gennes ${ }^{6}$ in 1967. The observations (i)-(iii) are also valid in this case. The long time behavior of

$\mathscr{S}(q, t)$ follows from equation $(82)$ as:

$$
\mathscr{S}(q, t) \rightarrow \exp \left[-1 \cdot 35(\Omega t)^{2 / 3}\right]
$$

where $\left(2^{2 / 3} / \pi\right) \Gamma(1 / 3) \simeq 1 \cdot 35$ has been used. Here $\Gamma(x)$ is the gamma function, and

$$
\int_{0}^{\infty} \mathrm{d} x\left(1-e^{-x^{3}}\right) / x^{3}=(1 / 2) \Gamma(1 / 3)
$$

In order to see how quickly the asymptotic time behaviour of $\mathscr{S}(q, t)$ given in equations (81) and (85) are attained, we have plotted $\log [-\ln \mathscr{S}(q, t)]$ vs. $\log \Omega(q) t$ in Figure 8 for $q a=0.3$. We observe that the asymptotic region is not reached in the experimental time range, i.e. $\Omega t \leqslant 10$. This implies that the exponents, which might be obtained by representing the experimental data by straight lines in the $\log$ - log plot, will be larger than the theoretical value $2 / 3$ in the Rouse-Zimm case, and $1 / 2$ in the Rouse limit.

We calculated $\mathscr{S}(q, t)$ from equation (78) which represents the asymptotic behaviour in the intermediate $q$-region and Rouse limit. The calculated points coincided exactly with the curve corresponding to $q a \leqslant 1$ in Figure 6. In the Rouse-Zimm case, we calculated $\mathscr{P}(q, t)$ using equation (82), and compared calculated points with the curve corresponding to $q a \leqslant 1$ in Figure 7 . The results agree within $2 \%$ or less, the estimates from equation
(82) being slightly larger. This discrepancy is probably due to the computational inaccuracies.

These two limiting cases actually follow from a more general expression for $\mathscr{S}(q, t)$ in the intermediate $q$ region:

$$
\mathscr{S}(q, t)=\int_{0}^{\infty} \mathrm{d} u \exp [-u-J(u, \Omega t, B / \sqrt{\alpha})]
$$

where

$$
\begin{aligned}
& J(u, \Omega t, B / \sqrt{\alpha}) \equiv \frac{2}{\pi} \int_{0}^{\infty} \mathrm{d} x \frac{\cos x u}{x^{2}}\{1- \\
& \left.\exp \left[-(\Omega t) x^{2} \frac{1+B(2 \pi / x \alpha)^{1 / 2}}{1+2 B(\pi / \alpha)^{1 / 2}}\right]\right\}
\end{aligned}
$$

This expression is derived from equation (73) in the limit of $\alpha \rightarrow 0$ but $(B / \sqrt{\alpha})$ finite, in order to discuss the effect of $B$, the draining parameter, on the shape of

$\mathscr{S}(q, t)$. The corresponding $\Omega(q)$ are easily obtained from equation (72b) with $\kappa \rightarrow \infty$ :

$$
\Omega(q)=\frac{1}{12}\left(k_{B} T / \xi_{0}\right) q^{4} a^{2}[1+(2 \sqrt{\pi} B / \sqrt{\alpha})]
$$

It is observed that $\mathscr{S}(q, t)$, in the intermediate $q$ region, is of the form $f(\Omega t, B / \sqrt{\alpha})$. The Rouse model (78) is recaptured from (86) with $B=0$. The Zimm limit corresponds, according to $(87)$ to $q a \ll 2 B(6 \pi)^{1 / 2}$. Since $B=0.38$ when the Flory value for the draining parameter $z_{H}=\left(\xi_{0} / \eta_{0} a\right)=5.2$ is used, this condition is satisfied when $q a \ll 3.2$.

Equation (86) indicates that $\mathscr{S}(q, t)$ approaches the Zimm limit asymptotically from below as $t \rightarrow \infty$, even $(B / \sqrt{\alpha}) 2 \sqrt{\pi}$ is not larger than unity. This follows from the fact that the main contribution to $J(u, \Omega t, B / \sqrt{\alpha})$ comes from the small values of $x$ in equation (86b) when $t$ is large. The cross over time, $t_{c}$, to the Zimm limit may be estimated qualitatively by equating the exponent in equation (86b) to unity when $B(2 \pi / \alpha x)^{1 / 2}$ $=1$ holds. This leads to:

$$
\Omega t_{c}=(1+2 \sqrt{\pi} z) / 8 \pi^{2} z^{4}
$$

where $z=B / \sqrt{\alpha}$. We deduce from equation (88) that $\Omega t_{c}$ varies from 0.09 to 17 , which may be considered as the experimentally accessible time range, when $B / \sqrt{\alpha}$ changes from $1 \cdot 0$ to $0 \cdot 2$, respectively. If we choose $\alpha$ $=0.1(q a \sim 0.77)$ as a typical value for $q a$ in the intermediate $q$ region, we find $B \simeq 0.3$ to $B \simeq 0.06$. Thus, the Zimm limit dominates in the time intervals of experimental interest when the Flory value $B=0.38$ is used. For times less than the cross over point, the behaviour of $\mathscr{S}(q, t)$ is determined by both Rouse and Zimm terms in the exponent of equation (86b). In general, the effect of $B$ on the shape of $\mathcal{P}(q, t)$, when it is expressed as a function of $\Omega t$, is such that $\mathscr{S}(q, t)$ increases with decreasing $B$, at a fixed $\Omega t$ and $q a$ (see Figure 9), from the Zimm value given by equation (82) to the Rouse value given by equation (78). 


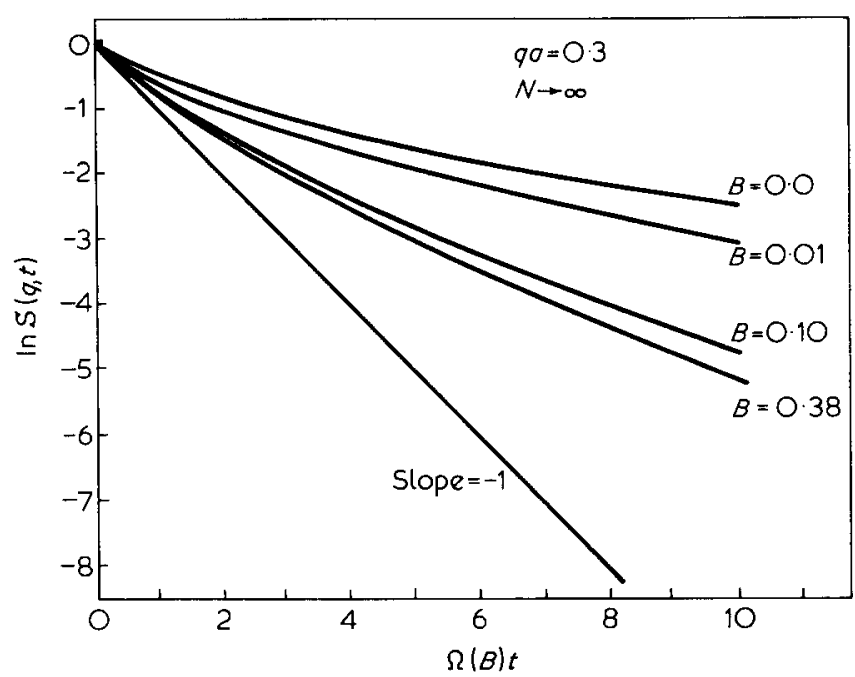

Figure 9 The effect of the draining parameter $B$ on the shape function $\ln S(q, t)$

Wang ${ }^{34}$ concluded from viscosity data that the draining parameter, $B$, may vary in the range 0.3 to 0.5 . In this region of values, the effect of $B$ on the shape function appears to be too small for $q a$ in the intermediate region to be of any practical significance. However, its effect is expected to be more pronounced when $q a$ is in the vicinity of unity. Since the value of $B$ is not known a priori at least with sufficient precision, it should be treated as an adjustable parameter in the interpretation of neutron scattering data where its effect is expected to be more important (see Figures in refs 10 and 43 ).

The asymptotic behaviour of $\mathscr{S}(q, t)$ for large values of $q(q a \gg 1)$ may be approximated by:

$$
\mathscr{S}(q, t) \approx \exp \left[-\alpha \varphi_{0}(t)\right]
$$

which follows from equation (73) keeping the first term only. Since the other terms contain a factor $\exp [-\alpha s]$, they decay faster for large $\alpha$ than equation (89). $\varphi_{0}(t)$ is defined by equation (74). It should be noted that

$\mathscr{S}(q, t)$ approaches Zimm behaviour and decays according to equation (85). After still larger times, it decays exponentially due to the translational diffusion. Depending on the $q$ value, one or several of these decay modes dominate the behaviour of $\mathscr{S}(q, t)$ during an experiment.

\section{DIRECT CALCULATION OF $\Omega$}

\section{General remarks}

It is possible to calculate $\Omega(q)$ directly using equation (11), when the dynamical operator, $\mathscr{L}$, and the equilibrium distribution function $\psi_{0}$ are given, even in cases where we may not be able to determine $\mathscr{P}(q, t)$ completely. The choice of $\mathscr{L}$ and $\psi_{0}$ in a given application depends on the variables used to characterize a state of the system, and on the model adopted to idealize the polymer.

Let $\mathscr{D}$ be chosen as the Kirkwood's generalized diffusion operator ${ }^{21}$. The adjoint of $\mathscr{D}$ is given by ${ }^{12}$

$$
\mathscr{L}=-\sum_{j, l=1}^{N}\left[\nabla_{j}+\left(\nabla_{j} \ln \psi_{0}\right)\right] \underline{\mathrm{D}}^{j l} \cdot \nabla_{l}
$$

The explicit form of $\mathrm{D}^{j l}$ and $\psi_{0}$ are not needed at this point. We assume that $\nabla_{j} \cdot \underline{\mathrm{D}}^{j l}=0$. Then, $\mathscr{L}$ has the property that, given two arbitrary $A$ and $B$ :

$$
\langle A, \mathscr{L} B\rangle=\sum_{j, l=1}^{N}\left\langle\nabla_{j} A^{*} \cdot \underline{\underline{D}}^{j l} \cdot \nabla_{l} B\right\rangle
$$

Using equation (91) in (11) yields:

$$
\Omega(q)=\left[\mathbf{q q}: \sum_{j, l=1}^{N}\left\langle e^{i \mathbf{q} \cdot \mathbf{R}_{j i} \underline{D}^{j l}}\right\rangle\right]\left[\sum_{j, l=1}^{N}\left\langle e^{i \mathbf{q} \cdot \mathbf{R}_{j i}}\right\rangle\right]^{-1}
$$

Equation (92) is the starting point in calculating $\Omega(q)$ for various chain models characterized by $\psi_{0}$, and for various models of the diffusion tensor. In this equation, the positions of the monomers, $\mathbf{R}_{j}$, are chosen as the variables to specify a state of the system, and $\psi_{0}$ is assumed to be a differentiable function of these variables. Consequently, equation (92) is readily applicable to problems involving flexible chains. In the case of chains with constraints, which can be described with fewer reduced variables, we must start with a modified dynamical operator, $\mathscr{L}$, involving only the reduced variables to avoid singularities in $\nabla_{j} \ln \psi_{0}$. Once $\mathscr{L}$ and $\psi_{0}$ in the reduced variables are agreed upon, we may proceed to calculate $\Omega(q)$ using equation (11). Since this point was somewhat unclear in a recent note $^{35}$, we shall first consider scattering from a rigid rod to illustrate the application of equation (11) to polymers with constraints.

\section{Single chain problem}

Scattering from a rigid rod. A state of a rigid rod is specified by $\mathbf{G}$ and $\Omega$, which denote the centre of mass and the orientation of the rod. These are the reduced variables of the problem. The distribution function $\psi(\mathbf{G}, \Omega, t)$ is assumed to satisfy ${ }^{36}$ :

$$
\dot{\psi}=\left(D_{T} \nabla_{G}^{2}-\Theta \hat{I}^{2}\right) \psi
$$

where $D_{T}$ and $\Theta$ are the translational and rotational diffusion coefficients respectively, and $I^{2}$ is the usual total angular momentum operator. The equilibrium distribution is uniform: $\psi_{0}(\mathbf{G}, \Omega)=(1 / 4 \pi V)$ where $V$ is the volume of the system. Since $\psi_{0}$ is constant, the adjoint operator $\mathscr{L}=-\mathscr{D}($ see equation 5$)$.

We assume that the rod contains $N$ equidistant scattering centres with a separation $b$. The length of the rod is $L=(N-1) b$. The density of scattering centres in Fourier space is:

$$
\rho(\mathbf{G}, \Omega)=e^{i \mathbf{q} \cdot \mathbf{G}} \sum_{n=1}^{N} e^{i \mathbf{q} \cdot \Omega b_{n}}
$$

where $b_{n}=[n-(N+1) / 2 b]$. Substitution of equations (93) and (94) into equation (11) yields:

$$
\Omega(q)=q^{2} D_{T}+\Theta \frac{\left\langle\rho, \hat{I}^{2} \rho\right\rangle}{\langle\rho, \rho\rangle}
$$

where the structure factor $\langle\rho, \rho\rangle$ can be calculated as

$$
\langle\rho, \rho\rangle=\sum_{l=0}^{\infty}(2 l+1)\left|\sum_{n=1}^{N} j_{l}\left(q b_{n}\right)\right|^{2}
$$


Equation (96) is obtained by expanding $\rho$ into spherical harmonics. Here $j_{l}(x)$ is the spherical Bessel function of order $l$. Since $\widehat{I}^{2} Y_{l m}=l(l+1) Y_{l m}$, we find $\Omega$ from equation (95) as:

$$
\Omega(q)=q^{2} D_{T}+\left[\Theta \sum_{l=0}^{\infty} l(l+1)(2 l+1)\left|\sum_{n=1}^{N} j_{l}\left(q b_{n}\right)\right|^{2}\right]\langle\rho, \rho\rangle^{-1}
$$

This result is identical to the initial slope of $\mathscr{S}(q, t)$ calculated directly by Pecora ${ }^{36}$, using the eigenfunction expansion method. The rigid dumb-bell result follows from equations (96) and (97) with $N=2$ and $b_{1}=-b / 2$ and $b_{2}=b / 2$ as:

$$
\Omega(q)=q^{2} D_{T}+\Theta \frac{x^{2}\left[(1 / 3)-j_{1}(x) / x\right]}{2\left[1+j_{0}(x)\right]}
$$

where $x=q L$. This result can also be obtained directly from equation (95) by calculating $\hat{I}^{2} \rho$ without expanding $\rho$ into spherical harmonics. When the random Langevin forces $\mathbf{F}_{j}(t), j=1,2$, acting on each of the monomers of the dumb-bell are uncorrelated, the translational and rotational diffusion coefficients can be expressed in terms of the diffusion coefficient, $D_{m}$ $=k_{B} T / \xi_{0}$, of a single monomer, as $D_{T}=D_{m} / 2$ and $\Theta=2 D_{m} / L^{2}$. Then (98) reduces to:

$$
\Omega(q)=q^{2} D_{m}\left[\frac{5}{6}+\frac{1}{2} j_{0}(x)-\frac{1}{x} j_{1}(x)\right]\left[1+j_{0}(x)\right]^{-1}
$$

When the Langevin forces are correlated, which is expected to be the case when the seperation of the monomers is less than the correlation length in the solvent, then $D_{T}=\left[D_{m}+D_{c}\right] / 2$ and $\Theta=2\left[D_{m}-D_{c}\right] / \mathscr{L}^{2}$, where $\left\langle\mathbf{F}_{j}(t) \mathbf{F}_{k}^{T}\left(t^{\prime}\right)\right\rangle=\underline{\underline{I}} 2 D_{j k} \delta\left(t-t^{\prime}\right), D_{11}=D_{22} \equiv D_{m}$, and $D_{12}=D_{21} \equiv D_{c}$ have been used ${ }^{37}$. We deduce from equation (99) that $\mathbf{\Omega}(q)=\left(D_{m} q^{2} / 2\right)$ as $q \mathscr{L} \rightarrow 0$, and $\Omega(q)=(5 / 6) D_{m} q^{2}$ as $q L \rightarrow \infty$. We shall comment on these results below.

Unperturbed Gaussian chain. (a) Without preaveraging Oseen tensor. Using equation (92), Akcasu and Gurol $^{10}$ obtained the following expression for $\Omega(q)$ (see equation 26 of this reference): with

$$
\mathscr{T}_{2}(q) \equiv(1 / 2)\left[-\alpha^{-1 / 2}+\left(2+\alpha^{-1}\right) \exp (-\alpha) \int_{0}^{\sqrt{\alpha}} \mathrm{d} u \exp \left(u^{2}\right)\right]
$$

In the small and large- $q$ limits, equation (100) yields, respectively, $\Omega(q)=\left(D_{m} q^{2} / 2\right)(1+B)$, and $\Omega(q)=D_{m} q^{2}$. At $\alpha=1(q a \sim 2 \cdot 45)$, which may be considered as a typical value in the intermediate $q$-region, one obtains $\Omega(q)$ $=D_{m} q^{2}(1+0.460 B) / 1.368$.

For analytical discussions of $\Omega(q)$ when $N$ is large, it is convenient to replace the summation in (100) by an integration, and use:

$$
\mathscr{T}_{N}(q)=\int_{\alpha}^{\kappa^{2}} \mathrm{~d} x\left(1-\frac{x}{\kappa^{2}}\right) \frac{1}{x}\left[\left(2+\frac{1}{x}\right) e^{-x} \int_{0}^{\sqrt{x}} \mathrm{~d} u e^{u^{2}}-\frac{1}{\sqrt{x}}\right]
$$

In the small- $q$ limit where $\kappa^{2} \ll 1$, equations (102) and (100) yield:

$$
\Omega(q)=\left(q^{2} D_{m} / N\right)[1+(8 / 3) B \sqrt{N}]
$$

The Zimm limit corresponds to the second term, i.e.:

$$
\Omega(q)=0 \cdot 195\left(k_{B} T / \eta_{0} a \sqrt{N}\right) q^{2}
$$

which is a well-known result ${ }^{14}$. In the intermediate- $q$ region where $\kappa^{2} \gg 1$ and $\alpha \ll 1$, we can show with $\alpha=0$ and $\kappa^{2} \rightarrow \infty$ that $\mathscr{T}_{N}=\pi \sqrt{\pi} / 2$. Hence, $\Omega(q)$ becomes

$$
\Omega(q)=q^{2} D_{m}(\alpha / 2)[1+(3 \pi \sqrt{\pi} / 4) B / \sqrt{\alpha}]
$$

which, in the Zimm limit, yields

$$
\Omega(q)=\frac{1}{16}\left(k_{B} T / \eta_{0}\right) q^{3}
$$

It is noted that the factor $(1 / 16)=0.0625$ in equation (106) was previously estimated as 0.055 by Akcasu and Gurol $^{10}$ (see equation 36 ) by approximating the

$$
\left.\Omega(q)=q^{2} D_{m}\left[1+\frac{3}{2} \frac{B}{\sqrt{\alpha}} \quad \mathscr{T}_{N}(q)\right]\left\{1+2\left(e^{\alpha}-1\right)^{-1}\left[1-\left(1-e^{-\kappa^{2}}\right) N^{-1}\left(1-e^{-\alpha}\right)^{-1}\right]\right\}^{-1} \quad(100 \mathrm{a})\right]
$$

where as before, $\kappa^{2}=q^{2} R_{g}^{2}$ and $\alpha=q^{2} a^{2} / 6$, and:

$$
\left.\mathscr{T}_{N}(q) \equiv \alpha \sum_{n=1}^{N-1}\left(1-\frac{n}{N}\right) X_{n}^{-2}\left[-X_{n}^{-1}+\left(2+X_{n}^{-2}\right) \exp \left(-X_{n}^{2}\right) \int_{0}^{x_{n}} \mathrm{~d} u \exp \left(\mathrm{u}^{2}\right)\right](100 \mathrm{~b})\right]
$$

Here $X_{n}^{2} \equiv n \alpha$.

We first calculate $\Omega(q)$ for a flexible dumb-bell in the presence of hydrodynamic interactions as a special case of equation (100) with $N=2$. The result is:

$\Omega(q)=\left[q^{2} D_{m}\left(1+e^{-\alpha}\right)^{-1}\right]\left[1+(3 / 2)(B / \sqrt{\alpha}) \quad \mathscr{T}_{2}(q)\right](101 \mathrm{a})$
Dawson integral in equation (102) by its series expansion. The exact factor in (106) was first calculated by Stockmayer ${ }^{38}$.

In the large- $q$ limit both $\alpha \gg 1$ and $\kappa^{2} \gg 1$ so that $\mathscr{F}_{N}(q) \rightarrow 0$ and $\Omega(q)=D_{m} q^{2}$, representing the diffusion of a single monomer.

(b) Preaveraging the Oseen tensor. The calculation of $\Omega(q)$ is greatly simplified if one replaces the diffusion 
matrix $\underline{D}^{j l}$ in $(92)$ by its preaveraged form. In this case, equation (92) reduces to:

$\Omega(q)=\left[\sum_{j, l=1}^{N}\left\langle e^{i \mathbf{q} \cdot \mathbf{R}_{\mu}}\right\rangle\left\langle\underline{\underline{D}}^{j l}\right\rangle: \mathbf{q} \cdot \mathbf{q}\right]\left[\sum_{j, l=1}^{N}\left\langle e^{\left.i \mathbf{q} \cdot \mathbf{R}_{j l}\right\rangle}\right]^{-1}\right.$

For a Gaussian chain $\left\langle\underline{\underline{D}}^{j l}\right\rangle$ is known to be ${ }^{14}$ :

$$
\left\langle\underline{\mathrm{D}}^{j l}\right\rangle=D_{m}\left[\delta_{j l}+\left(1-\delta_{j l}\right) \frac{B}{\sqrt{[j-l \mid}}\right] \stackrel{I}{=}
$$

Substitution of equation (108) into (107) yields an expression for $\Omega(q)$ similar to $(100)$, in which $\mathscr{T}_{N}(q)$ now reads:

$$
\mathscr{\mathscr { F }}_{N}(q)=(4 \sqrt{\alpha} / 3) \sum_{n=1}^{N-1}\left(1-\frac{n}{N}\right) \frac{1}{\sqrt{n}} \exp (-n \alpha)
$$

The Gaussian dumb-bell result follows from equations (101a) and (109) with $\mathscr{T}_{2}(q)=(2 \sqrt{\alpha} / 3) \exp (-\alpha)$. Comparison of the latter with (101) indicates that the use of the preaveraged Oseen tensor does not affect $\Omega(q)$ in the small $(\alpha \ll 1)$ and large- $q$ regions. At $\alpha=1$ $(q a \sim 2.45)$ we find $\Omega(q)=D_{m} q^{2}(1+0.368 B) / 1.368$ which is slightly smaller than the value obtained previously without preaveraging.

When $N \gg 1, \mathscr{\mathscr { T }}_{N}(q)$ in equation (109) can be approximated by an integral as

$$
\mathscr{T}_{N}(q)=\frac{4}{3} \int_{\alpha}^{\kappa^{2}} \cdot \mathrm{d} x\left(1-\frac{x}{\kappa^{2}}\right) \frac{1}{\sqrt{x}} \exp (-x)
$$

which may be expressed in terms of incomplete gamma functions if desired. Comparing (110) and (102), and verifying $\mathscr{T}_{N}(\kappa \rightarrow 0)=(16 / 9) \kappa$ and $\mathscr{T}_{N}(\alpha \rightarrow \infty)=0$ in equation (110), we conclude that the preaveraging does not affect $\Omega(q)$ in the small- and large- $q$ regions. In the intermediate- $q$ region, equation (110) yields, with $\alpha \rightarrow 0$ and $\kappa^{2} \rightarrow \infty, \quad \mathscr{T}_{N}(q)=4 \pi^{1 / 2} / 3$ so that we obtain, in the Zimm limit, $\Omega(q)=\left(k_{B} T / \eta_{0}\right)(1 / 6 \pi) q^{3}$. This result is identical to equation (84) which was obtained from the full expression of $\mathscr{S}(q, t)$ using the preaveraged Oseen tensor. Comparison with equation (106), however, shows that the preaveraging underestimates $\Omega(q)$ by a factor of 0.848 in the intermediate- $q$ region. This trend prevails for all $q$ values in the transition region from the intermediate- to large- $q$ values, becoming more pronounced when $q a$ approaches unity, as shown by Akcasu and Gurol ${ }^{10}$ by computing $\Omega(q)$ numerically using equations (100) and (109).

Burchard and his coworkers calculated $\Omega(q)$ using equation (92) for polydisperse linear chains ${ }^{39}$, regular and polydisperse star-macromolecules ${ }^{40}$, randomly and non-randomly branched polycondensates ${ }^{41}$ and randomly crosslinked chains ${ }^{42}$. Recently, they also investigated the effect of preaveraging the Oseen tensor on $\Omega(q)$ for branched polymers, and concluded that the error due to preaveraging may be as high as $40 \%$ in such polymers ${ }^{42}$.
Unperturbed freely jointed chains. In order to include stiffness into the model, Akcasu and Higgins ${ }^{43}$ calculated $\Omega(q)$ for a freely jointed chain, again starting from equation (92). Except for some slight changes in symbols, their result reads:

$\Omega(q)=q^{2} D_{m}\left[\frac{N+1}{N}+\left(\frac{3}{8 \pi}\right)^{1 / 2} B \mathscr{F}_{N}(\kappa)\right]\left[\frac{N+1}{N}+2 \mathscr{G}_{N}(\kappa)\right]^{-1}$

where $N$ is the number of bonds, $\kappa \equiv \mathrm{qb}$ and

$$
\begin{aligned}
& \mathscr{F}_{N}(\kappa) \equiv \int_{0}^{\infty} \mathrm{d} x \quad x \kappa \quad \mathscr{G}_{N}(\kappa x)\left[\left(1+x^{2}\right) \ln \left|\frac{1+x}{1-x}\right|-2 x\right] \\
& \mathscr{G}_{N}(\kappa) \equiv \frac{j_{0}(\kappa)}{1-j_{0}(\kappa)}\left[1-\frac{j_{0}(\kappa)}{1-j_{0}(\kappa)} \frac{1-j_{0}^{N}(\kappa)}{N}\right]
\end{aligned}
$$

In (111) we have not introduced the usual approximation $N+1 \sim N$ for large $N$, as done in reference (43), because here we also wish to calculate $\Omega(q)$ for a rigid dumb-bell as a special case of equation (111), with $N$ $=1$. The result is:

$$
\Omega(q)=q^{2} D_{m}\left[1+j_{0}(\kappa)\right]^{-1}\left[1+(3 \pi / 8)^{1 / 2} B\left(j_{0}(\kappa)-\kappa^{-1} j_{1}(\kappa)\right)\right]
$$

In the small- $q$ limit $(114 a)$ yields $\Omega(q)=\left(D_{m} q^{2} / 2\right)[1$ $\left.+(\pi / 6)^{1 / 2} B\right]$ which is slightly smaller than the value obtained for the Gaussian dumb-bell using (101). In the large- $q$ limit $\Omega(q)=D_{m} q^{2}$, both for the rigid and Gaussian dumb-bells. However, this result is different from that obtained from equation (99), i.e. $\Omega(q)$ $=D_{m} q^{2}(5 / 6)$, in which the bond constraint was introduced in the dynamical operator, $\mathscr{L}$, at the outset, This discrepancy has been pointed out recently in a note by Stockmayer et al. ${ }^{35}$. We think that the introduction of the constraint in $\mathscr{L}$ at the outset, and including it at the end through $\psi_{0}$ as the limit of a peaked intermonomer potential correspond to two different physical dynamical models, and thus yield two different results. In the former, the relaxation time, $T_{b}$, associated with bond-length variations [e.g. $T_{b}$ $\sim\left(\xi_{0} / K\right)$ when the interaction between monomers is represented by a harmonic potential with a spring constant $K$ is assumed to be smaller than the correlation time, $\tau$, of the random Langevin forces acting on the beads. Hence, the results correspond to taking the limits $T_{b} \rightarrow 0$ and $\tau \rightarrow 0$, in this order. In the latter model, it is tacitly assumed that $T_{b}>\tau$, and the results correspond to the limit $\tau \rightarrow 0$ and then $T_{b} \rightarrow 0$ at the end. Depending on the physical application, one may choose one of these two models. The predictions of these two models differ from each other appreciably only in the large- $q$ region, where chain models for the real polymer, based on an effective bond length $b$ and associated friction coefficient $\xi_{0}$ per segment, e.g. freely-jointed chain model, are bound to fail eventually. Since $b$ and $\xi_{0}$ are two adjustable parameters in such models, the discrepancy mentioned above may be 


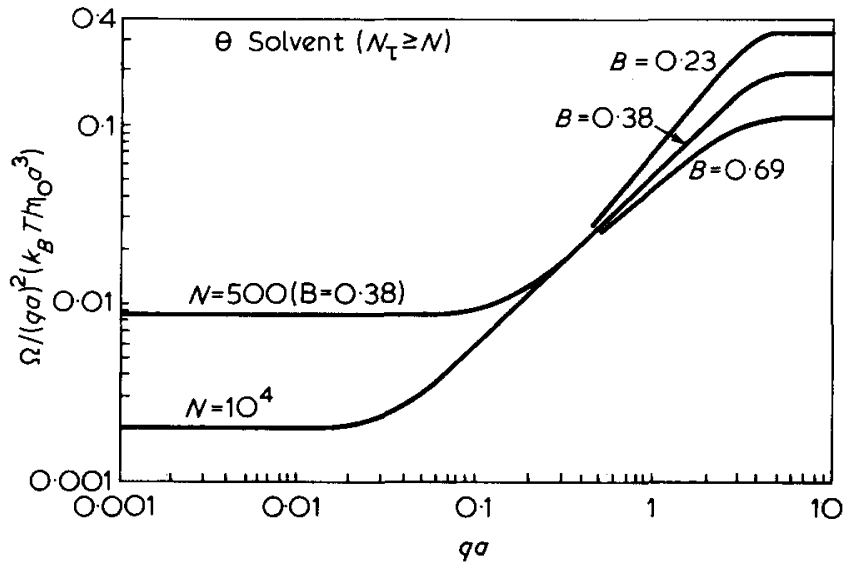

Figure 10 The variation of the initial slope, $\Omega(q)$, with $(q a)$ under $\theta$-condition for various values of the draining parameter $B$, and two values of $N$. Note that the curves for different $B s$ and a fixed $N$ coincide at small values of $q a$

attributed to different choices of $b$ and $\xi_{0}$ in the interpretation of experiments in large- $q$ regions.

The variation of $\Omega(q)$ with $\kappa$ when $N \gg 1$ was investigated by Akcasu and Higgins ${ }^{41}$ for various values of the draining parameter $\left(\xi_{0} / \eta_{0} a\right)$ using equations (111) and (112). In the small- $q$ limit, we find $\Omega(q)$ $=D(N) q^{2}$ where the diffusion coefficient $D(N)$ is given by:

$$
D(N)=D_{m} N^{-1}\left[1+(8 / 3 \pi)^{1 / 2} B \int_{0}^{\infty} \mathrm{d} x \mathscr{G}_{N}(x)\right]
$$

The integral in equation (114b) approaches to $2 \cdot 8931 \sqrt{N}$ so that one obtains $D(N)$ $=0 \cdot 195\left(k_{B} T / b \eta_{0} \sqrt{N}\right)$ in the Zimm limit which is identical to the result equation (104) for a Gaussian chain. The freely jointed and Gaussian chain models predict different results mainly for large values of $q$ where $q b$ is in the vicinity of unity. For large values of $q(q b \gg 1)$, equation (111) yields $\Omega(q)=D_{m} q^{2}$ as in the case of Gaussian chain, which corresponds to the diffusion of a single bead.

Excluded volume effects. The excluded volume effects can be included in the calculation of $\Omega(q)$ using the basic formulae (92) or (107). This was done by Benmouna and $\mathrm{Akcasu}^{44}$ for a single chain with preaveraged Oseen tensor using equation (107). Calculation of $\Omega(q)$ in the case of a single chain involves only the equilibrium distribution of the vector distance $\mathbf{R}_{j l}$ between $j$ th and $l$ th monomers belonging to the same chain. Benmouna and $\mathrm{Akcasu}^{44}$ approximated the characteristic function of this distribution as

$$
\exp \left[\frac{-q^{2}}{6}<\left|\mathbf{R}_{j l}\right|^{2}>\right]
$$

in which $\left\langle\left|\mathbf{R}_{n}\right|^{2}\right\rangle$ is modelled using the blob model of chain statistics $^{8 y}$, i.e.:

$$
\begin{array}{ll}
\left\langle\left|\mathbf{R}_{n}\right|^{2}\right\rangle=n a^{2}, & \text { for } n \leqslant N_{\tau} \\
=\left(n / N_{\tau}\right)^{2 v} \xi_{\tau}^{2}, & \text { for } n \geqslant N_{\tau}
\end{array}
$$

where $n=|j-l|$ and $\xi_{\tau}^{2}=N_{\tau} a^{2} . N_{\tau}$ is the number of monomers within a temperature blob, and is related to the reduced temperature $\tau=(T-\theta) / T$ by $N_{\tau} \sim \tau^{-2}$. The proportionality constant in the latter is not specified at this stage. The blob model implies that the excluded volume interaction becomes important only when the chemical distance $n$ exceeds $N_{\tau}$. The temperature effects are included in the calculation of $\Omega(q)$ through $N_{\tau}$. The diffusion tensor, $\mathrm{D}^{j l}$ must be averaged with respect to the above distribütion function, i.e.,

$$
\begin{aligned}
& 1\left\langle\underline{\mathrm{D}}^{j l}\right\rangle=\underline{\underline{\mathrm{I}}} D_{m}\left[\delta_{j l}+\left(1-\delta_{j l}\right) B(|j-l|)^{-1 / 2}\right], \quad|j-l| \leqslant N_{\tau} \\
& =\underline{\underline{\mathrm{I}} D_{m}}\left[\delta_{j l}+\left(1-\delta_{j l}\right) B N_{\tau}^{v-1 / 2}(|j-l|)^{-v}\right], \quad|j-l| \geqslant N_{\tau}
\end{aligned}
$$

Substitution of equation (116) into (107) yields after replacing summations by integration $(N \gg 1)$ :

$$
\Omega(q)=q^{2} D_{m}[1+2(B / \sqrt{\alpha}) \quad \mathscr{G}(\kappa, \tau)][1+2 H(\kappa, \tau) / \alpha]^{-1}
$$

where

$$
\begin{array}{r}
\mathscr{G}(\kappa, t) \equiv \int_{\alpha}^{x \kappa^{2}} \mathrm{~d} u\left(1-u / \kappa^{2}\right) u^{-1 / 2} \exp (-u)+ \\
\left(x \kappa^{2}\right)^{v-1 / 2} \int_{x \kappa^{2}}^{\kappa^{2}} \mathrm{~d} u\left(1-u / \kappa^{2}\right) u^{-v} \exp \left[-u^{2 \nu}\left(x \kappa^{2}\right)^{1-2 v}\right]
\end{array}
$$

and

$$
\begin{array}{r}
H(\kappa, \tau) \equiv \int_{\alpha}^{x \kappa^{2}} \mathrm{~d} u\left(1-u / \kappa^{2}\right) e^{-u}+ \\
\int_{x \kappa^{2}}^{\kappa^{2}} \mathrm{~d} u\left(1-u / \kappa^{2}\right) \exp \left[-u^{2 v}\left(x \kappa^{2}\right)^{1-2 v}\right]
\end{array}
$$

In these equations $\kappa^{2}=\alpha N, \alpha=q^{2} a^{2} / 6$ and $x \equiv N_{\tau} / N$. Both $G$ and $H$ can be expressed in terms of incomplete gamma functions ${ }^{44}$. The good and $\theta$-solvent limits can both be obtained from (117) by letting $x=N^{-1}$ and $x$ $=1$, respectively. In the latter case, we reproduce the results presented above. If we let $v=1 / 2$ in (117), we again recapture the $\theta$-solvent limit.

Figures 10 and 11 show the variation of $\Omega(q)$ as a function of $(q a)$ for various values of the draining parameter, $B$, in $\theta$-solvent and good solvent conditions, respectively. The curves are calculated using the original expression ${ }^{44}$ for $\Omega(q)$ before summations are replaced by integrals, rather than equation (117). The approximation of replacing summations by integrals results in slightly larger $\Omega(q)$, but always less than $10 \%$, the largest discrepancies occurring in the vicinity of qa $=1$. The computer results are presented in Tables 3 and 4.

In the small- $q$ limit, equation (117) yields the temperature dependence of the diffusion coefficient $D(T)$ $=\lim _{q \rightarrow 0} \Omega(q) / q^{2}$ : 
Interpretation of dynamic scattering from polymer solutions: A. Ziya Akcasu et al.

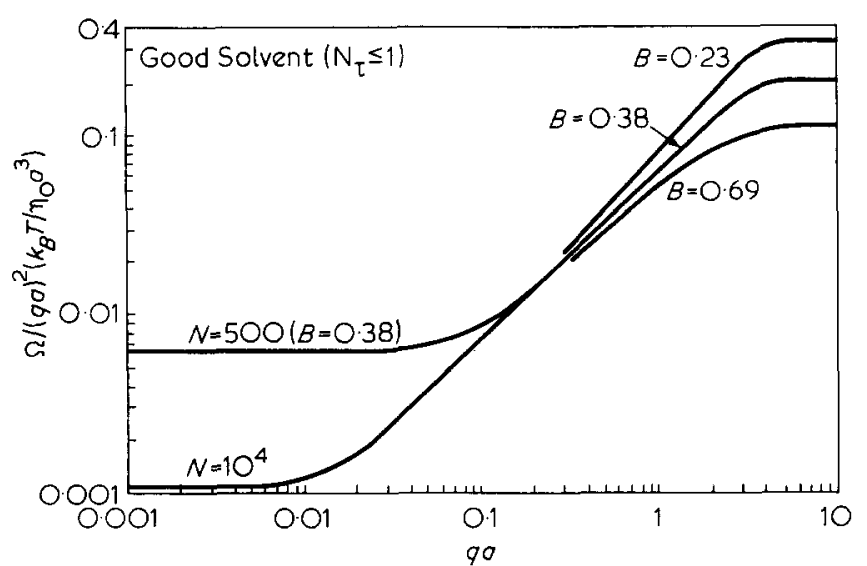

Figure 11 The variation of the initial slope, $\Omega(q)$ with $(q a)$ in a good solvent for various values of the draining parameter $B$, and two values of $N$. Note that the curves for different $B$ s and a fixed $N$ coincide at small values of $q a$

Tab/e 3 Normalized characteristic frequency $\Omega / q^{2}\left(k_{B} T / \xi_{0}\right)$ versus qa in $\theta$ solvent $\left(N_{\tau} \geqslant N\right)$ for various $Z_{H} \equiv\left(\xi_{0} / \eta_{0} a\right)=\pi \sqrt{6 \pi B}$

\begin{tabular}{|c|c|c|c|c|}
\hline$Z_{H}$ & 0 & $\pi$ & $1.65 \pi$ & $3 \pi$ \\
\hline 0.001 & 0.0001 & 0.00619 & 0.0101 & 0.0183 \\
\hline 0.002 & 0.0001 & 0.00619 & 0.0101 & 0.0184 \\
\hline 0.003 & 0.0001 & 0.0062 & 0.0101 & 0.0184 \\
\hline 0.004 & 0.0001 & 0.0062 & 0.0102 & 0.0184 \\
\hline 0.005 & 0.0001 & 0.0062 & 0.0102 & 0.0184 \\
\hline 0.006 & 0.0001 & 0.0062 & 0.0102 & 0.0185 \\
\hline 0.007 & 0.0001 & 0.0062 & 0.0102 & 0.0185 \\
\hline 0.008 & 0,0001 & 0.0063 & 0.0103 & 0.0186 \\
\hline 0.009 & 0.0001 & 0.0063 & 0.0103 & 0.0187 \\
\hline 0.01 & 0.0001 & 0.0063 & 0.0104 & 0.0187 \\
\hline 0.02 & 0.0001 & 0.0067 & 0.0110 & 0.020 \\
\hline 0.03 & 0.0001 & 0.0075 & 0.0122 & 0.022 \\
\hline 0.04 & 0.0002 & 0.0085 & 0.0138 & 0.025 \\
\hline 0.05 & 0.0003 & 0.0097 & 0.0158 & 0.028 \\
\hline 0.06 & 0.0004 & 0.0111 & 0.0181 & 0.033 \\
\hline 0.07 & 0.0005 & 0.0126 & 0.0205 & 0.037 \\
\hline 0.08 & 0.0006 & 0.0142 & 0.0231 & 0.041 \\
\hline 0.09 & 0.0007 & 0.0158 & 0.0257 & 0.046 \\
\hline 0.1 & 0.0009 & 0.0175 & 0.0283 & 0.051 \\
\hline 0.2 & 0.0034 & 0.0347 & 0.0550 & 0.097 \\
\hline 0.3 & 0.0075 & 0.0527 & 0.0820 & 0.143 \\
\hline 0.4 & 0.0134 & 0.0713 & 0.109 & 0.187 \\
\hline 0.5 & 0.0209 & 0.0904 & 0.135 & 0.230 \\
\hline 0.6 & 0.0300 & 0.110 & 0.162 & 0.270 \\
\hline 0.7 & 0.0409 & 0.130 & 0.188 & 0.309 \\
\hline 0.8 & 0.0533 & 0.151 & 0.215 & 0.347 \\
\hline 0.9 & 0.0675 & 0.173 & 0.241 & 0.383 \\
\hline 1 & 0.0832 & 0.195 & 0.267 & 0.418 \\
\hline 2 & 0.3220 & 0.447 & 0.528 & 0.696 \\
\hline 3 & 0.6350 & 0.713 & 0.764 & 0.869 \\
\hline 4 & 0.8700 & 0.899 & 0.918 & 0.958 \\
\hline 5 & 0.969 & 0.976 & 0.981 & 0.990 \\
\hline 6 & 0.995 & 0.996 & 0.997 & 0.998 \\
\hline 7 & 0.999 & 1.0 & 1.0 & 1.0 \\
\hline 8 & 1.0 & 1.0 & 1.0 & 1.0 \\
\hline 9 & 1.0 & 1.0 & 1.0 & 1.0 \\
\hline 10 & 1.0 & 1.0 & 1.0 & 1.0 \\
\hline
\end{tabular}

$D(T)=D(\theta) \frac{3}{4} x^{1 / 2}\left[2\left(1-\frac{x}{3}\right)+\frac{1}{1-v}\left(x^{v-1}-1\right)-\frac{1}{2-v}\left(x^{v-1}-x\right)\right]$

where $D(\theta)$ denotes the diffusion coefficient at $\theta$ temperature, i.e.:

$$
D(\theta)=(8 / 3 \pi \sqrt{6 \pi})\left(k_{B} T / \eta_{0} a \sqrt{N}\right)
$$

In both (118) and (119) we have ignored the Rouse term [see equations (103) and (104)].

In the good solvent limit $D(T)$ becomes

$$
D(\operatorname{good})=[2 / \pi \sqrt{6 \pi}(1-v)(2-v)]\left(k_{B} T / \eta_{0} a N^{v}\right)
$$

Akcasu and $\mathrm{Han}^{45}$ used equation (118) to define a temperature dependent hydrodynamic radius $R_{H}(T)$ as:

$$
R_{H}(T)=\left[k_{B} T / 6 \pi \eta_{0} D(T)\right]
$$

They investigated the temperature dependence of the linear expansion factor, $\alpha_{H}(T) \equiv R_{H}(T) / R_{H}(\theta)$. The proportionality constant in $N_{\tau}=$ constant $/ \tau^{2}$ was determined from the data on $\alpha_{s}(T) \equiv R_{g}(T) / R_{g}(\theta)$, where the temperature dependence of the radius of gyration $R_{g}$ was obtained using the blob model, equation (115). They studied the variation of $\alpha_{H}(T)$ as a function of $\left(N / N_{\tau}\right)$ that combines both the molecular weight and temperature dependences. $\mathrm{Han}^{4 C}$ used the above expression for $R_{H}(T)$ and $R_{g}(T)^{45}$ and calculated the intrinsic viscosity from $[\eta(T)] /[\eta(\theta)]$ $=R_{g}^{2}(T) R_{H}(T) / R_{g}^{2}(\theta) R_{H}(\theta)^{47}$ as a function of $\left(N / N_{\tau}\right)$. He showed that the viscosity data agrees well with the theoretical predictions.

Table 4 Normalized characteristic frequency $\Omega / q^{2}\left(k_{B} T / \xi_{0}\right)$ versus qa in a good solvent $\left(N_{\tau} \geqslant 1\right)$ for various $Z_{H}=\left(\xi_{0} / \eta_{0} a\right)=\pi \sqrt{6 \pi} B$

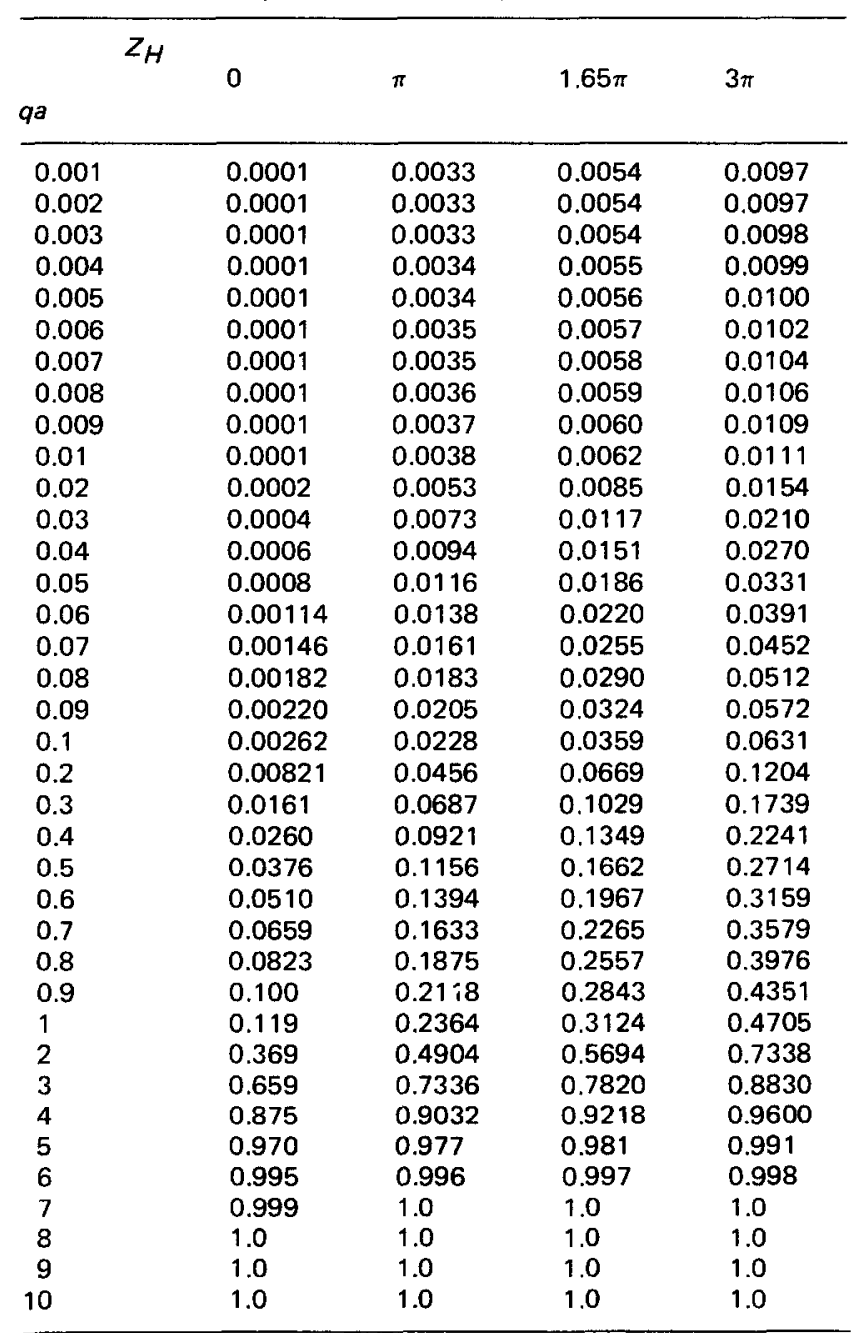


The intermediate $q$-values of $\Omega(q)$ is obtained from equation (117) with $\alpha \rightarrow 0$ and $\kappa^{2} \rightarrow \infty$. Depending on the value of $x \kappa^{2}=\left(q^{2} \xi_{\tau}^{2} / 6\right)$ relative to unity either the first or second term dominates in equations (117b) and $(117 \mathrm{c})$. Thus, $\Omega(q)$ displays a cross over behaviour at a temperature-dependent momentum transfer $q_{\tau}^{*}=6^{1 / 2} / \xi_{\tau}$ :

and

$$
\Omega(q)=0.053\left(k_{B} T / \eta_{0}\right) q^{3} ; \quad \text { for } q \geqslant q_{\tau}^{*}
$$

$$
\Omega(q)=0.071\left(k_{B} T / \eta_{0}\right) q^{3}, \quad \text { for } q \leqslant q_{\tau}^{*}
$$

In the good solvent limit, $\xi_{\tau}=a$, and $q_{\tau}^{*}$ is outside the intermediate $q$-region. Thus, equation (123b) represents the asymptotic behaviour of $\Omega(q)$ in a good solvent for the intermediate values of $q$. In the case of nonpreaveraged Oseen tensor, the coefficient 0.071 in (123 b) becomes 0.079 as shown by Benmouna and Akcasu recently ${ }^{51}$.

The large- $q$ limit of (117) yields $\Omega(q)=\left(k_{B} T / \xi_{0}\right) q^{2}$ with $\alpha \rightarrow \infty$ and $\kappa^{2} \rightarrow \infty$.

Single labelled chains. Here we calculate $\Omega(q)$ for a single labelled chain, such as a deuterated chain in the presence of protonated chains in a good solvent, as function of concentration. We use equation (107) in which the concentration dependence is introduced through the equilibrium distribution of $\mathbf{R}_{j l}$ using the blob model:

$$
\begin{aligned}
\left\langle\left|\mathbf{R}_{n}\right|^{2}\right\rangle & =a^{2} n^{2 v} ; \quad \text { for } n \leqslant N_{c} \\
& =\left(n / N_{c}\right) \xi_{c}^{2} ; \quad \text { for } n \geqslant N_{c}
\end{aligned}
$$

where $\xi_{c}=a N_{c}^{\nu} \sim c^{-3 / 4}$. Using equation (124) in (107) Akcasu and Benmouna ${ }^{48}$ obtained $\Omega(q)$ as follows:

$$
\Omega(q)=q^{2} D_{m}\left[1+2 B \alpha^{(v-1) / 2 v} \mathscr{G}(\kappa, c)\right]\left[1+2 \alpha^{-1 / 2 v} \mathscr{H}(\kappa, c)\right]^{-1}
$$

which yields the correct single chain diffusion coefficient in the zero concentration limit with $x=1$ :

$$
D(0)=[2 / \pi \sqrt{6 \pi}(1-v)(2-v)]\left[k_{B} T / \eta_{0} a N^{v}\right]
$$

This is, of course, identical to equation (120). In the semidilute region where $N \gg N_{c} \gg 1$, the second term in equation (125) dominates so that:

$$
D(\text { semidilute })=0 \cdot 195\left[k_{B} T / \eta_{0} \xi_{c} \sqrt{N / N_{c}}\right]
$$

which corresponds to the diffusion coefficient of a Gaussian chain with $\left(N / N_{c}\right)$ blobs each of size $\xi_{c}$.

In the intermediate $q$-range, the variation of $\Omega(q)$ with $q$, as calculated from (125) with $\alpha=0$ and $\kappa^{1 / v}$ $=\infty$, also shows ${ }^{48}$ a crossover behaviour at $q^{*}=6^{1 / 2} \xi_{c}$ in the intermediate $q$-range similar to that in the temperature case. Akcasu and Benmouna ${ }^{48}$ discussed also the effect of screening of the hydrodynamic interaction on the $q$-dependence of $\Omega(q)$. Since the dynamic operator, $\mathscr{L}$, does not include the entanglement effects, the above results are not applicable to the cases where such effects are important.

\section{Scattering from identical chains}

In the cse of scattering from identical chains, the expression for $\Omega(q)$ in equation (92) can be written as $^{48}$ :

$$
\begin{gathered}
\Omega(q)= \\
\frac{\mathrm{qq}: \sum_{n, m=1}^{N}\left[\left\langle\underline{\mathrm{D}}^{1 n, 1 m} e^{i \mathbf{q} \cdot\left(\mathbf{R}_{1 n}-\mathbf{R}_{1 m}\right)}\right\rangle+\left(N_{p}-1\right)\left\langle\underline{\underline{D}}^{1 n, 2 m} e^{i \mathbf{q} \cdot\left(\mathbf{R}_{1 n}-\mathbf{R}_{2 m}\right)}\right\rangle\right]}{\sum_{n, m=1}^{N}\left[\left\langle e^{i \mathbf{q} \cdot\left(\mathbf{R}_{1 n}-\mathbf{R}_{1 m}\right)}\right\rangle+\left(N_{p}-1\right)\left\langle e^{i \mathbf{q} \cdot\left(\mathbf{R}_{1 n}-\mathbf{R}_{2 m}\right)}\right\rangle\right]}
\end{gathered}
$$

where

$$
\mathscr{G}(\kappa, c) \equiv \int_{\substack{\alpha^{2 v} \\ \alpha^{1 / v}}} \mathrm{~d} u\left(1-\frac{u}{\kappa^{1 / v}}\right) u^{-v} \exp \left(-u^{2 v}\right)+\left(x \kappa^{1 / v}\right)^{(1-2 v) / 2} \int_{x \kappa^{1 / v}}^{\kappa^{1 / v}} \mathrm{~d} u\left(1-\frac{u}{\kappa^{1 / v}}\right) u^{-1 / 2} \exp \left[-u\left(x \kappa^{1 / v}\right)^{2 v-1}\right]
$$

and

$$
\mathscr{H}(\kappa, c) \equiv \int_{\alpha^{1 / 2 v}}^{x \kappa^{1 / v}} \mathrm{~d} u\left(1-\frac{u}{\kappa^{1 / v}}\right) e^{-u^{2 v}}+\int_{x \kappa^{1 / v}}^{\kappa^{1 / v}} \mathrm{~d} u\left(1-\frac{u}{\kappa^{1 / v}}\right) \exp \left[-u\left(x \kappa^{1 / v}\right)^{2 v-1}\right]
$$

Here $\kappa^{2}=\alpha N^{2 v}$ and $x \equiv N_{c} / N$. The dilute and concentrated solution limits can be obtained from equation (125) by letting $x \equiv 1$ and $x=(1 / N)$, respectively. For example, equation (125) with $x \equiv 1$ is identical to equation (117) with $x=1 / N$.

From the small- $q$ limit of equation (125) we obtain the concentration dependent diffusion coefficient in the Zimm limit as:

$$
\begin{aligned}
D(c) & =\frac{2}{\pi \sqrt{6 \pi}} \frac{k_{B} T}{\eta_{0} a N^{v}} x^{1-v}\left[\left(\frac{1}{1-v}-\frac{x}{2-v}\right)+\right. \\
& \left.\frac{2}{x^{1 / 2}}\left(\frac{2}{3}-\sqrt{x}+\frac{x^{3 / 2}}{3}\right)\right]
\end{aligned}
$$

where $N_{p}$ is the number of chains in the solution. The second sum in both the numerator and denominator of equation (129) involves the distribution of the vector distance between monomers belonging to two different chains designated by 1 and 2 in the subscripts of $\mathbf{R}_{1 n}$ and $\mathbf{R}_{2 m}$. Due to the difficulty in modelling this distribution, Akcasu and Benmouna ${ }^{48}$ approximated the distance $\left|\mathbf{R}_{1 n}-\mathbf{R}_{2 m}\right|$ by the distance between the centre of masses of the two polymers, i.e. $\left|\mathbf{R}_{1}-\mathbf{R}_{2}\right|$. They modelled the intermolecular interaction by a hard sphere potential with a radius $\bar{S}$ which is expressed in terms of the second virial coefficient ${ }^{14}$. This simplifying assumption is justified away from the $\theta$-temperature where interpenetration of molecules is 
less significant due to excluded volume interaction. Considering the small- $q$ limit of equation (129) they calculated the concentration dependence of $D(T, c)$ in the dilute region as:

$$
D(T, c)=D(T, 0)\left[1+c k_{D}\right]
$$

where $D(T, 0)$ is given by equation $(118), c$ is the concentration in volume fraction with a volume $\left(4 \pi R_{H}^{3} / 3\right)$ per polymer molecule, and $k_{D}$ is given by:

$$
k_{D}(T, N)=\left(\bar{S} / R_{H}\right)^{2}\left[8\left(\bar{S} / R_{H}\right)-6\right]
$$

Here $R_{H}$ is related to $D(T, 0)$ by equation (121). Among other things, equation (131) showed that $k_{D}$ changes sign when $\bar{S}=0.75 R_{H}$, implying that $D(T, c)$ decreases with concentration in poor solvents and increases in good solvents. The above theoretical analysis will be extended to $\theta$-conditions, and compared with experiment in a separate paper ${ }^{49}$.

\section{INTERPRETATION OF SCATTERING EXPERIMENTS}

\section{Light scattering experiments}

Quasielastic light scattering experiments were carried out with a full-photon counting Rayleigh spectrometer $^{50}$. A $4880 \AA$ line from a Ar-Ion laser was used as the light source. Momentum transfer $q$ is selected through scattering angle, $\theta$. In this case, $\theta$ ranges from about $10^{\circ}$ to $150^{\circ}$ through goniometer arrangement

A polystyrene sample of $48 \times 10^{6}$ from Japan Synthetic Rubber Company with $M_{W} / M_{n} \sim 1.3$ was kindly given to us by Professor J. Ferry from the University of Wisconsin. Toluene was used as the solvent with a polymer concentration of $0.22 \mathrm{mg} / \mathrm{ml}^{-1}$. This concentration is much lower than the concentration $c^{*}$ of semidilute region. All experiments were carried out at room temperature, $T=23^{\circ} \mathrm{C}$.

Since homodyne beating configuration was used, the normalized scattering function $\mathscr{S}(q, \tau)$ is obtained from the normalized experimental photon correlation function, $C(q, \tau)$ through

$$
\mathscr{S}^{2}(q, \tau)=\frac{C(q, \tau)-1}{\beta}
$$

where $\beta$ is related to the efficiency and was treated as an adjustable parameter. This introduces an extra parameter as a constant in all the following analyses, but will not be mentioned again for the sake of convenience. For a weak scattering polymer system, $\beta$ is usually around $0 \cdot 1$, therefore, one would expect a noise level of at least $1 \%$ in $\mathscr{S}(q, t)$ for a measurement of $\Delta C / C \sim 2 \times 10^{-3}$. In many cases, the noise-tosignal ratio is worse than $1 \%$. The experimental results will be presented in the following section as part of the data analysis.

\section{Interpretation of the data}

We assume that $\mathscr{S}(q, t)$ is measured by light scattering as explained above, or by neutron scattering with spin-echo tehnique, and the results presented as ln $\mathscr{S}(q, t)$ vs. time for each value of $q$ used in the experiment. Our method of interpretation consists of fitting a theoretical shape function $f\left(\Omega t, q a, q R_{g}, B\right)$ to the experimental data by adjusting $\Omega, B$ and $a$ or $R_{g}$, and then comparing the experimental $\Omega(q)$ with its thoretical expression.

Light scattering. In the light scattering experiments qa is much less than unity so that the only relevant characteristic size of the chain is $R_{g}$. For such small values of $q a$ the shape function is insensitive to the values of the draining parameter (see equation 87 and the subsequent discussions) and $N$. In fact it depends only on $\tau=\Omega t$ and $\kappa=q R_{g}$ for finite $\kappa$ and $N \gg 1$ and $q a \ll 1$. Indeed, substituting $t=\tau / \Omega$ and taking the limit of $N \rightarrow \infty$ with fixed $\kappa=q R_{g}$, we obtain $\mathscr{S}(q, t)$ from equation (71) as function of $\kappa$ and $\tau$ :

$$
\begin{gathered}
\mathscr{P}(q, t)=2 P^{-1}(\kappa) e^{-\tau / F(\kappa)} \int_{0}^{1 / 2} \mathrm{~d} y \exp \left\{-2 \kappa^{2}[y(1-y)+\right. \\
\left.\left.\frac{1}{\pi^{2}} \sum_{k=1}^{\infty} \frac{\cos 2 \pi k y}{k^{2}}\left(1-\exp \left\{-\tau \frac{2 \pi k^{2}}{\kappa^{2} F(\kappa)} \int_{0}^{1 / 2} \mathrm{~d} x \frac{\cos 2 \pi k x}{\sqrt{x(1-x)}}\right\}\right)\right]\right\}
\end{gathered}
$$

where $P(\kappa)$ is defined in equation (47b) and:

$$
F(\kappa) \equiv P^{-1}(\kappa)\left[I_{0}\left(\kappa^{2} / 4\right) \exp \left(-\kappa^{2} / 4\right)\right]
$$

We may cast equation (133a) into a more convenient form by replacing the $k$ summation by an integral with the trapezoidal approximation:

$$
\mathscr{S}(q, t)=\left[\kappa^{2} P(\kappa)\right]^{-1} \int_{0}^{\kappa^{2}} \mathrm{~d} u \exp \left[-u\left(1-\frac{u}{2 \kappa^{2}}\right)-h\left(u, \kappa^{2}\right)\right]
$$

where

$$
\begin{gathered}
h\left(u, \kappa^{2}\right) \equiv \frac{2}{\pi} \int_{0}^{\infty} \mathrm{d} x \frac{\cos x u}{x^{2}}[1- \\
\left.\exp \left\{-\tau\left(x^{2} / \kappa F(\kappa)\right) \frac{\sqrt{2}}{\pi} \int_{0}^{\kappa^{2}} \mathrm{~d} y \frac{\cos x y}{\left[y\left(1-y / 2 \kappa^{2}\right)\right]^{1 / 2}}\right\}\right]
\end{gathered}
$$

one can verify as a check that $\mathrm{d} \mathscr{S}(q, t) / \mathrm{d} \tau=-1$ is satisfied by both expressions. We observe from equation (133c) that $\mathscr{S}(q, t)$ approaches its asymptotic behaviour in the intermediate- $q$ region (see equation 82) when $\kappa \rightarrow \infty$. The large-time behaviour of $\mathscr{S}(q, t)$ readily follows from equation (133a) as:

$$
\mathscr{S}(q, t)=P^{-1}(\kappa) e^{-\kappa^{2} / 3} e^{-\tau / F(\kappa)}
$$

which represents the decay of the translational diffusion mode (compare equation 133e to the first term in equation 43$)$. In obtaining equation (133e) we have used $^{26}$ : 
Interpretation of dynamic scattering from polymer solutions: A. Ziya Akcasu et al.

$$
\sum_{k=1}^{\infty} \frac{\cos k x}{k^{2}}=\frac{\pi^{2}}{6}-\frac{\pi x}{2}+\frac{x^{2}}{4}(0 \leqslant x \leqslant 2 \pi)
$$

The draining parameter drops out as a result of the limit $N \rightarrow \infty$. It is interesting to point out that $F(\kappa)$ defined in equation (133b) is the ratio of the initial slope to the decay constant of the diffusive mode for a single Gaussian closed chain in a $\theta$-solvent, i.e. $F(\kappa)$ $\equiv \Omega(q) / D_{\text {ring }} q^{2}$ where $\Omega(q)$ is given in equation $(72 b)$. This ratio for an open chain both in good and $\theta$-solvents is given in equation (134).

We have plotted the variation of the shape function $f\left(\Omega t, q R_{g}\right) \equiv \ln \mathscr{S}(q, t)$ in Figure 4 using equation (71) directly with $B=0.38$ and $N=101$. These values of $B$ and $N$ have no significance because the results are insensitive to them provided that $N \gg 1$. We observe that the shape function attains its asymptotic form very closely when $q R_{q} \geqslant 4 \cdot 35$. For values of $q R_{g}$ larger than $4 \cdot 35$, we may switch to the infinite chain shape function in the intermediate- $q$ region, which is presented in Figure 7. For the smaller values of $q R_{g}$, the curves presented in Figure 4 or the corresponding analytical formulas given in equation (133) provide an interpolation between the above asymptotic behaviour and the straight line reached as $q R_{g} \rightarrow 0$. This procedure enables us to interpret the data in this transition as well as in the asymptotic regions.

In order to determine $\Omega(q)$ and $R_{g}$ simultaneously by fitting $f\left(\Omega t, q R_{g}\right)$ to the experimental points, we suggest the following iteration procedure.

(i) Fit a polynomial of the form:

$$
f\left(\Omega t, q R_{g}\right)=-(\Omega t)\left[1+A_{1}(\Omega t)+A_{2}(\Omega t)^{2}+\ldots\right]
$$

to $\ln \mathscr{S}(q, t)$ data (or any cumulant fit) and obtain $\Omega(q)$ as a function of $q$, as a first estimate. Our experience show that a polynomial of fifth order is needed to increase the accuracy of this estimate when $q R_{g} \geqq 4$. We mention in passing that the expansion coefficients in this polynomial are related to the higher cumulants. We also note that we do not use any a priori knowledge of the shape function in this step;

(ii) In order to estimate $R_{g}$, compare the experimental $\Omega(q)$ obtain in (i) with an appropriate theoretical expression of $\Omega(q)$ corresponding to the conditions of the experiment and the chain model adopted to describe the actual polymer molecule. These theoretical formulae have been presented in above. In our experiment, we treated toluene as a good solvent and used equation (117) with $N_{\tau}=1$. In the limit of $q a \rightarrow 0$, equation (117) reduces to

$$
\frac{\Omega(q)}{q^{2} D(v)}=\frac{(1-v)(2-v)}{2} \kappa_{v} \frac{\gamma\left(\frac{1-v}{2 v}, \kappa_{v}^{2}\right)-\kappa^{-1 / v} \gamma\left(\frac{2-v}{2 v}, \kappa_{v}^{2}\right)}{\gamma\left(\frac{1}{2 v}, \kappa_{v}^{2}\right)-\kappa_{v}^{-1 / v} \gamma\left(\frac{1}{v}, \kappa_{v}^{2}\right)}
$$

where

$$
\begin{aligned}
\kappa_{v}^{2} & \equiv(1 / 3)(1+v)(1+2 v) q^{2} R_{g}^{2}(v) \\
D(v) & \equiv \frac{k_{B} T}{\eta_{0} R_{g}(v)}\left\{\pi(1-v)(2-v)[3 \pi(1+v)(1+2 v)]^{1 / 2}\right\}^{-1}
\end{aligned}
$$

$$
R_{g}(v) \equiv a N^{v}[2(1+v)(1+2 v)]^{-1 / 2}
$$

and where $\gamma(\mu, x)$ denotes incomplete gamma function defined by:

$$
\gamma(\mu, x) \equiv \int_{0}^{x} \mathrm{~d} t t^{\mu-1} e^{-t}
$$

We note that (134) yields $\Omega(q)$ also in $\theta$ condition with $v=1 / 2$. For solvents in between for which $1<N_{\tau}<N$ one must use the original equation (117) with $x=N_{\tau} / N$.

The $\Omega(q)$ in equation (134) depends only on $R_{g}$ when the temperature and viscosity of the solvent are given. By fitting equation (134) to the measured $\Omega(q)$, one obtains a first estimate of $R_{g}$. This procedure makes use of all the experimental points to extract $R_{q}$.

(iii) The accuracy of the first estimate of $\Omega(q)$ can be improved by fitting the experimental $\mathscr{S}(q, t)$ for each $q$, to the theoretical $\mathscr{S}(q, t)=\exp \left[f\left(\Omega t, q R_{g}\right)\right]$ in equation (133). In this curve fitting procedure we use in $f\left(\Omega t, q R_{g}\right)$ the first estimate of $R_{g}$ obtained in (ii), and treat $\Omega(q)$ as an adjustable parameter. The new improved value of $\Omega(q)$ is then used in (ii) to obtain an improved estimate for $R_{g}$. The use of closed chain results to predict the shape function in the transition region is perhaps somewhat crude, but at present we do not have a tractable expression for $\mathscr{S}(q, t)$ for open chains in the Rouse-Zimm model (see equation 67). By way of justifying the use of equation (133), we mention that the closed chain shape function approaches to the asymptotic behaviour for an infinite chain given by equation (82) closely when $q R_{g} \sim 4 \cdot 35$, and exactly when $q R_{g} \rightarrow \infty$. It also reproduces the single exponential decay as $q R_{g} \rightarrow 0$.

Figure 12 shows the experimental values of $\ln \mathscr{S}(q, t)$ as function of $\Omega(q) t$, and the corresponding theoretical shape functions for the $q$-values used in the experiment. The $\Omega(q)$ values used for $q R_{g} \geqslant 3 \cdot 23$ were obtained after the first iteration, i.e. after step (ii). We note that all of the data points for $\Theta \geqslant 23^{\circ}$ fall on top of each other within experimental accuracy indicating that asymptotic intermediate- $q$ behaviour has already

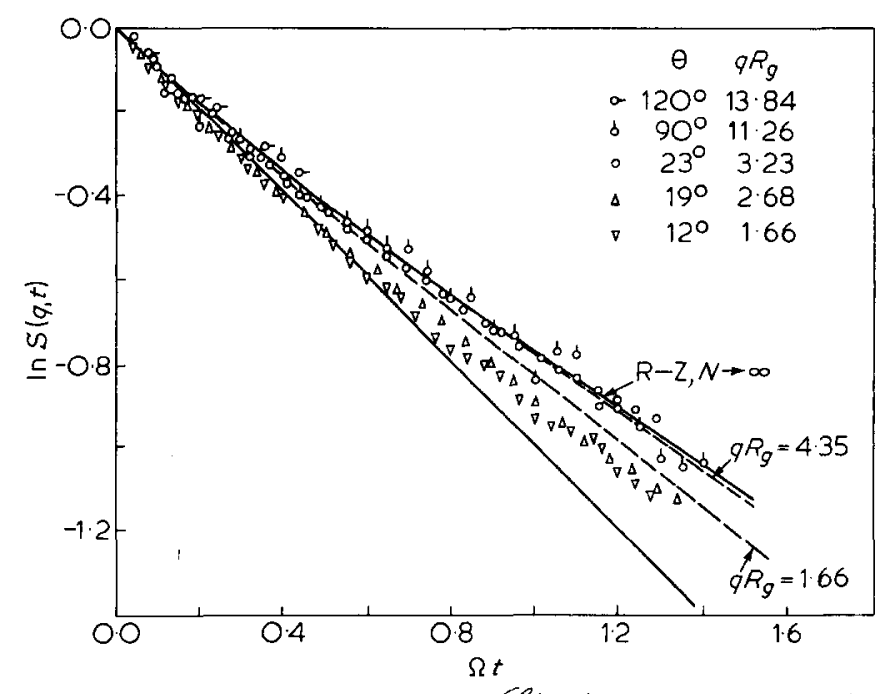

Figure 12 Experimental values of $\ln \mathscr{S}(q, t)$ as a function of $(\Omega \tau)$ for various scattering angles $\theta$ for polystyrene in toluene 


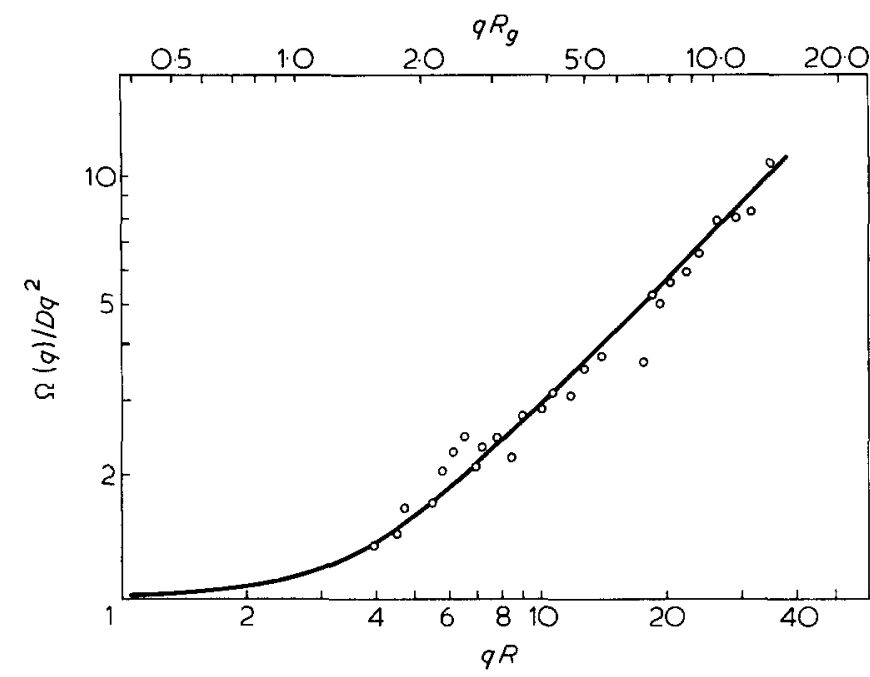

Figure 13 Comparison of the experimental and theoretical values of $\Omega(q)$. Polystyrene $M=48 \times 10^{6}$ in toluene; $R=1.1 \times 10^{-4} \mathrm{~cm}$; $R_{g}=4.14 \times 10^{-5} \mathrm{~cm}$

been reached. The solid curve marked $(R-Z, N \rightarrow \infty)$ denotes this asymptotic limit as calculated from equation (82) (see also Figure 7). The dashed curve was obtained from equations (71) and (72) with $q R_{g}=4 \cdot 35$. The data points corresponding to $q R_{g}=2.68$ and 1.66 are plotted using $\Omega(q)$ as calculated from the polynomial fit in the zeroth iteration, i.e. after step (i). We also plotted the shape function calculated from equations (71) and (72) with $q R_{g}=1 \cdot 66$, for comparison. The values of $\Omega(q)$ at these $q$ values have not been adjusted to match the experimental points and the above shape function, because we intend to compare the results obtained for $\Omega(q)$ with the polynominal fit and the shape function before adjustment.

Figure 13 compares the experimental and theoretical $\Omega(q)$. The latter was calculated using equation (134) with $R_{g}=4.14 \times 10^{-5} \mathrm{~cm}$. Had we had adjusted the values of $\Omega(q)$ for $q R_{g}=2.68,1.66$ and 1.50 , the lowest three points in this Figure would have been raised slightly. This adjustment would have resulted in about $5-10 \%$ increase in $R_{g}$. An independent estimate of the radius of gyration for polystyrene with $M=48 \times 10^{6}$ by the procedure of Akcasu and $\mathrm{Han}^{45}$ is $4.50 \times 10^{-5}$ $\mathrm{cm}$. The agreement is highly satisfactory particularly in view of the fact that our experiment was not originally designed to measure $R_{g}$ accurately. Our interest was to demonstrate how a light scattering experiment can be interpreted even in the transition region where the asymptotic laws are not valid. Information about $R_{g}$ is contained in the $q$ region where the $\Omega(q)$ vs. $q$ curve bends towards its horizontal asymptote. We would measure $\mathscr{S}(q, t)$ in the latter region if the primary purpose of the experiment had been to determine $R_{g}$ accurately.

\section{Neutron scattering}

The interpretation of neutron scattering experiments follows the same procedure as outlined above for light scattering. In (i) we obtain the first estimate of $\Omega(q)$ again by a polynomial fit to $\ln \mathscr{S}(q, t)$ data. In (ii) we compare the experimental and theoretical $\Omega(q)$ to determine the effective segment length $a$ and the associated draining parameter $B$. For dilute solutions in the good solvent limit, equation (117) yields with $\kappa^{2}$ $\rightarrow \infty$ and $x=1 / N$ :

$$
\frac{\Omega(q)}{q^{2} D_{m}}=\frac{1+B\left[\alpha^{(v-1) / 2 v} / v\right]\left[\Gamma\left(\frac{1-v}{2 v}\right)-\gamma\left(\frac{1-v}{2 v}, \alpha\right)\right]}{1+\left[\alpha^{-1 / 2 v} / v\right]\left[\Gamma\left(\frac{1}{2 v}\right)-\gamma\left(\frac{1}{2 v}, \alpha\right)\right]}
$$

where $\alpha=q^{2} a^{2} / 6$, and $\Gamma(x)$ is the complete gamma function. This expression is valid under $\theta$-conditions also with $v=1 / 2$. Figures 10 and 11 show the variation of $\Omega(q)$ with $q a$ for several values of $B$. We note that $\Omega(q)$ depends only on $a$ and $B$ when $k_{B} T$ and $\eta_{0}$ are known. Hence, with a two-parameter curve-fitting procedure to the experimental $\Omega(q)$ one obtains the first estimates for $a$ and $B$. In step (iii) we improve the values of $\Omega(q)$ by comparing the experimental $\ln \mathscr{S}(q, t)$ and the theoretical shape function calculated from equation (73) using the first estimates of $a$ and $B$. Finally, we update the values of $a$ and $B$ repeating step (ii). Figure 7 depicts $f(\Omega t, q a, B)$ with $B$ $=0.38$, as an example. The asymptotic behaviour given in equation (82) persists for all $q a \leqslant 1$. For these values of $q a$, the shape function depends only on $(\Omega t)$, and hence, the iteration procedure described above may be skipped when $q a \ll 1$ holds.

It should be emphasized that the spring-bead model is bound to fail for large values of $q a$ where the details, such as the bond-length, bond-angle constraints, steric hinderances, play an important role. We have presented freely-jointed chain models for calculating $\Omega(q)$ in step (ii), as a somewhat improved model in the sense that the stiffness effects are taken into account at least qualitatively in terms of a fixed bond length $b$. Clearly more realistic models are needed to interpret neutron scattering data for such large- $q$ values. But it is still interesting to investigate the predictions of these models, and to see how far they are able to explain the trends in the experimental data with adjustable parameters $a$ (or $b$ ) and $B$.

In spite of these difficulties in their interpretation, the neutron scattering experiments in the upper transition region where $q a$ approaches unity, have the potential of providing information on the effective bond length and the associated friction coefficient per segment (or draining parameter), and as such are interesting both from theoretical and experimental point of view.

\section{DISCUSSION}

In this paper we presented a method of interpretation of light and neutron scattering experiments in terms of the initial slope $\Omega(q)$ of the normalized intermediate scattering function, $\mathscr{S}(q, t)$. The reason for choosing $\Omega(q)$, which is also called the first cumulant of $\mathscr{P}(q, t)$, as a basis for the interpretation of data is that it can be calculated as a function of temperature and concentration of the solution, for all values of $q$, even in cases where $\mathscr{P}(q, t)$ itself is not calculable.

Furthermore, $\Omega(q)$ contains information about the chain parameters such as the effective bond length, friction coefficient per segment, and radius of gyration. The 
main shortcoming of the proposed method of interpretation is that $\Omega(q)$ is determined from $\mathscr{S}(q, t)$ data using a shape function which is calculated for a single unperturbed Gaussian chain. The crucial assumption here is that the shape function is less sensitive than $\Omega(q)$, to temperature and concentration effects. Until an accurate theoretical calculation of $\mathscr{S}(q, t)$ including temperature and concentration effects becomes possible, the proposed procedure may serve as a reasonable interim method of interpretation of scattering experiments. It is emphasized, however, that the above assumption is needed only in determining $\Omega(q)$ experimentally from $\mathscr{S}(q, t)$ data, and thus affects only the accuracy of the measurement of $\Omega(q)$. Neither the meaning nor the theoretical evaluation of $\Omega(q)$ relies on this assumption. The use of an a priori known shape function provides a better accuracy than the polynomial or cumulant fit to extract $\Omega(q)$ from the experimental data.

The concentration and temperature effects on $\Omega(q)$ are taken into account by modelling $\psi_{0}\left(\mathbf{R}_{i j}\right)$, i.e. the equilibrium distribution of the vector distance between the $i$ th and the $j$ th monomers, using blob model of chain statistics. However, the expression

$$
\Omega(q)=\frac{\left\langle\rho^{*} \mathscr{L} \rho\right\rangle}{\left\langle\rho^{*} \rho\right\rangle}
$$

for $\Omega(q)$ is quite general, and can accommodate other equilibrium models for $\psi_{0}\left(\mathbf{R}_{i j}\right)$. The method of interpretation proposed in this paper is clearly not based on the blob model. Furthermore, the dynamical operator $\mathscr{L}$ is taken to be the Kirkwood-Riseman diffusion operator, but other model operators can also be used in the calculation of $\Omega(q)$.

[Refer to note added in proof at the end of the paper.]

\section{ACKNOWLEDGEMENTS}

The authors express their gratitude for fruitful discussions during the progress of this work with Drs A. Peterlin, E. DiMarzio, I. Sanchez and C. Guttman at the National Bureau of Standards. Acknowledgement is made to the donors of Petroleum Research fund, administered by the American Chemical Society for partial support of Mustapha Benmouna and A. Ziya Akcasu. The first author also acknowledges the summer support received from the SANS program of the National Bureau of Standards.

\section{REFERENCES}

1 For example: (a) Chu, B. 'Laser Scattering', Academic Press, NY, 1974; (b) Photon Correlation and Light Beating Spectroscopy (Ed. H. Z. Cummins and E. R. Pike) Plenum Press, NY, 1974; (c) Berne, B. J. and Pecora, R. Dynamic Light Scattering, Wiley, NY, 1976

2 Richter, D. et al. Phys, Rev. Lett. 1978, 41, 1484

3 Pecora, R. J. Chem. Phys. 1965, 43, 1562

4 Pecora, R. J. Chem. Phys. 1968, 49, 1032

5 de Gennes, P. G. Physics 1967, 3, 37

6 Dubois-Voilette, E. and de Gennes, P. G. Physics 1967, 3, 181

7 Adam, M. and Delsanti, M. Macromolecules 1977, 10, 1229

8 Daoud, M. Thesis, Universite de Paris 1977

9 Farnoux, B. et al. Physics, 1978, 39, 77

10 Akcasu, A. Z. and Gurol, H. J. Polym. Sci. (Polym. Phys. Edn.) 1976, 14, 1

11 Zwanzig, R. J. Chem. Phys. 1974, 60, 2717
12 (a) Fixman, M. J. Chem. Phys. 1965, 42, 3831

(b) Pyun, C. W. and Fixman, M. J. Chem. Phys. 1965, 42, 3838

13 des Cloizeaux, J. CEN (Saclay) Reports 1976

14 For example: Yamakawa, H. "Modern Theory of Polymer Solutions', Harper and Row, NY 1971

15 Bixon, M. J. Chem. Phys. 1972, 58, 1459

16 Zwanzig, R. 'Lectures in Theoretical Physics' (Eds W. E. Brittin, W. B. Downs and J. Downs) Wiley, NY 1961, Vol. 3, p. 106

17 Mori, H. Prog. Theor. Phys. 1965, 33, 423

18 (a) Mori, H. Prog. Theor. Phys. 1965, 34, 399

(b) Jhon, M. S., Fesciyan, S. and Dahler, J. S. J. Polym. Sci. (to be published)

19 Kapral, R. et al. J. Chem. Phys. 1976, 64, 539

20 Akcasu, A. Z. and Duderstadt, J. J. 'Kinetic Equations' (Ed. R. L. Liboff and N. Rostoker) Gordon and Breach Science, NY, 1971

21 Riseman, J. and Kirkwood, J. G. Rheology, Theory and Applications, Academic Press, NY, 1956

22 Wang, M. C. and Uhlenbeck, G. E. Rev. Mod. Phys. 1945, 17, 323

23 Shore, J. E. and Zwanzig, R. J. Chem. Phys. 1975, 63, 5445

24 Bellman, R. 'Introduction to Matrix Analysis', McGraw-Hill, NY

25 Rouse, P. E. Jr. J Chem. Phys. 1953, 21, 1272

26 Handbook of Mathematical Functions, (Eds M. Abramowitz and I. A. Stegun) AMS 555, NBS 1964. Also, Tables of Integrals, Series and Products, by I. S. Gradshteyn and I. W. Ryzhik, Academic Press, New York 1965.

27 Zimm, B. H. J. Chem. Phys. 1956, 24, 269

28 Bloomfield, V. A. and Zimm, B. H. J. Chem. Phys. 1966, 44, 315

29 Burchard, W., Stockmayer, W. H. and Schmidt, M. personal communication

30 Flory, P. J. 'Principles of Polymer Chemistry', Cornell University Press, Ithaca, NY, 1953, pp. 609, 623

31 Zwanzig, R. et al. Proc. Natl. Acad. Sci. 1968, 60, 381

32 Ullman, R. Macromolecules, 1974, 7, 300

33 Fong, J. T. and Peterlin, A. J. Res. NBS-B, Math. Sci. 1976, 80B, 273

34 Wang, F. W. J. Polym. Sci. (Polym. Phys. Edn.) 1975, 13, 1215

35 Stockmayer, W. H. and Burchard, W. J. Chem. Phys. 1979, 70, 3138

36 (a) Pecora, R. J. Chem. Phys. 1964, 40, 1604

(b) Pecora, R. J. Chem. Phys. 1968, 48, 4126

37 The details of the calculation will be presented elsewhere

38 Stockmayer, W. H. personal communication

39 Burchard, W. Macromolecules, 1978, 11, 455

40 Stockmayer, W. H., Schmidt, M. and Burchard, W. Macromolecules 1980, 13, 580

41 Schmidt, M. and Burchard, W. Macromolecules 1978, 11, 460

42 Burchard, W., Schmidt M. and Stockmayer, W. H. 1979 Macromolecules (in press)

43 Akcasu, A. Z. and Higgins, J. S. J. Polym. Sci. (Polym. Phys. Edn), $1977,15,1745$

44 Benmouna, M. and Akcasu, A. Z. Macromolecules 1978, 11, 1187

45 Akcasu, A. Z. and Han, C. C. Macromolecules 1979, 12, 276

46 Han, C. C. Polymer 1979, 20, 1083

47 Weill, G. and des Cloizeaux, J. Physics 1979, 40, 99

48 Akcasu, A. Z. and Benmouna, M. Macromolecules 1978, 11, 1193

49 Han, C. C. and Akcasu, A. Z. (to be published)

50 (a) Han, C. C. Rev. Sci. Instrum. 1978, 49, 31

(b) Han, C. C. Polymer 1979, 20, 259

51 Benmouna, M. and Akcast, A. Z. Macromolecules 1980, 13, 409

52 Fixman, M. Personal communication (to be published)

53 Horta, A. and Fixman, M. J. Am. Chem. Soc. 1968, 90, 3048

\section{APPENDIX A}

\section{Derivation of equation (29)}

Since the $x$-axis is parallel to $\mathbf{q},\left\langle v_{m}, \rho\right\rangle$ is given by

$$
\left\langle v_{m}, \rho\right\rangle=\sum_{j=1}^{N} \prod_{k=2}^{N} \int_{-\infty}^{+\infty} \mathrm{d} x\left(\frac{3 \mu_{k}}{2 \pi a^{2}}\right)^{1 / 2} e^{-\left(3 \mu_{k} / 2 a^{2}\right) x^{2}} v_{m_{k}}(x) e^{i q Q_{j k} x}
$$

where $v_{m_{k}}(x)$ is given by (26). The crucial integral 
needed in (A1) is:

$\int_{-\infty}^{+\infty} \mathrm{d} x H_{m}(x) \exp \left[-x^{2}+i \alpha x\right]=(i \alpha)^{m} \sqrt{\pi} \exp \left(-\alpha^{2} / 4\right)$

which can be proven by combining 7.388.1 and 7.388.3 on p. 840 of ref 26 . Substitution of (26) and (A2) into (A1) yields:

$\left\langle v_{m}, \rho\right\rangle=\sum_{j=1}^{N} \prod_{k=2}^{N}\left(m_{k} ! 2^{m_{k}}\right)^{-1 / 2}\left(i q a \sqrt{2 / 3 \mu_{k}} Q_{j k}\right)^{m_{k}} e^{-\left(q^{2} a^{2} / 6 \mu_{k}\right) Q_{j k}^{2}}$

This leads to

$$
\begin{aligned}
& \left|\left\langle v_{m}, \rho\right\rangle\right|^{2}= \\
& \sum_{j, l=1}^{N} \prod_{k=2}^{N} \frac{1}{m_{k} !}\left(\frac{q^{2} a^{2}}{3 \mu_{k}} Q_{j k} Q_{l k}\right)^{m_{k}} \exp \left[-\frac{q^{2} a^{2}}{6 \mu_{k}}\left(Q_{j k}^{2}+Q_{l k}^{2}\right)\right]
\end{aligned}
$$

(29) is obtained from (A4) by allowing the matrix elements of $Q_{j k}$ to be complex as in the case of a ring polymer.

In order to obtain (30) from (27) we only need to observe the following identity for arbitrary $\left(Z_{1}, \ldots Z_{N}\right)$ :

$$
\sum_{m_{1}, \ldots, m_{N}=0}^{\infty} \prod_{l=1}^{N}\left(Z_{l}\right)^{m_{l} / m_{l} !}=\sum_{n=0}^{\infty} \frac{1}{n !}\left[\sum_{l=1}^{N} Z_{l}\right]^{n}
$$

\section{APPENDIX B}

\section{Ring polymer calculations}

The crucial step in obtaining equation (44) is to show that

$$
\sum_{j, l=1}^{N} e^{-\alpha \varphi_{i j-I}(t)}=\mathrm{N}\left[e^{-\alpha \varphi_{0}(t)}+2 \sum_{s=1}^{N-1}\left(1-\frac{s}{N}\right) e^{-\alpha \varphi_{j}(t)}\right]
$$

where $\varphi_{s}(t)$ is given in equation (45b). Verifying that $\varphi_{s}$ $=\varphi_{-s}$ and $\varphi_{s+k N} \equiv \varphi_{s}$ for $k=0, \pm 1, \ldots$ we can prove:

$$
\sum_{s=1}^{N-1}\left(1-\frac{s}{N}\right) e^{-\alpha \varphi_{s}} \equiv \frac{1}{2} \sum_{s=1}^{N-1} e^{-\alpha \varphi_{s}}
$$

When $N=2 K+1$, the second term is equal to $\Sigma_{S}^{K} \exp [$ $\left[-\alpha \varphi_{s}\right]$, and equation (44) follows.

The form in equation (48) is obtained by first verifying:

$\varphi_{s}(t)=\varphi_{s}(0)+\int_{0}^{2} \frac{W t}{\mathrm{~d} x} e^{-x} \frac{1}{N} \sum_{k=1}^{N-1} e^{x \cos (2 \pi k / N)} \cos (2 \pi k s / N)$

Then, using:

$$
\exp [x \cos z]=\sum_{n=-\infty}^{+\infty} I_{n}(x) \cos n z
$$

and

$\sum_{k=1}^{N-1} \cos \left[\frac{2 \pi}{N}(n \pm s) k\right]=\left\{\begin{array}{l}(N-1), \text { if } n \pm s=m N, m=0, \pm 1, \ldots \\ -1, \text { otherwise }\end{array}\right.$

we obtain after several steps:

$$
\varphi_{s}(t)=\psi_{s}(t)-\frac{2 W t}{N}
$$

where $\varphi_{s}(t)$ is given in equation (48b). Note that the second term in (B6) removes the factor $\exp \left[-D_{m} q^{2} t / N\right]$ when (B6) is substituted into equation (44), and (48a) follows.

\section{APPENDIX C}

Summation formulae

Some of the following summation formulae have been used in the text:

$\frac{1}{2 N} \sum_{m=1}^{N-1} \frac{\sin ^{2}(m s \pi / N)}{\sin ^{2}(m \pi / 2 N)}=|s|\left(1-\frac{|s|}{N}\right), \quad|s|=0,1, \ldots, N$

$\frac{1}{2 N} \sum_{m=1}^{N-1} \frac{\sin ^{2}(m s \pi / N)}{\cos ^{2}(m \pi / 2 N)}=|s|\left(1-\frac{|s|}{N}\right), \quad|s|=0,1, \ldots, N$

$\frac{1}{N} \sum_{m=1}^{N-1} \frac{\sin ^{2}(m s \pi / N)}{\sin ^{2}(m \pi / N)}=|s|\left(1-\frac{|s|}{N}\right), \quad|s|=0,1, \ldots, N$

$$
\begin{aligned}
& \frac{1}{2 M} \sum_{m=1}^{M-1} \frac{\sin ^{2}(m s \pi / 2 M)}{\sin ^{2}(m \pi / 2 M)}=\frac{1}{2}\left[|s|\left(1-\frac{|s|}{2 M}\right)-\right. \\
&\left.\frac{\sin ^{2}(s \pi / 2)}{2 M}\right], \quad|s|=0,1, \ldots, 2 M
\end{aligned}
$$

$I(N) \equiv \sum_{m=1}^{N-1} \sin ^{-2}(m \pi / N)=\left(N^{2}-1\right) / 3$

$$
\begin{gathered}
\frac{1}{2 N} \sum_{m=1}^{N-1} \frac{\left[\cos \left(s-\frac{1}{2}\right) \frac{m \pi}{N}-\cos \left(t-\frac{1}{2}\right) \frac{m \pi}{N}\right]^{2}}{\sin ^{2}(\pi m / 2 N)}=|s-t|, \\
s, t=1,2, \ldots, N
\end{gathered}
$$

The formula (C1) follows from the more general identity $\frac{1}{2 N} \sum_{m=1}^{N-1} \frac{\sin (m s \pi / N) \sin (m t \pi / N)}{\sin ^{2}(m \pi / 2 N)}=$

$$
\frac{|s+t|-|s-t|}{2}-\frac{s t}{N}, \quad s, t=1,2, \ldots, N
$$

We found equation (C7) accidentally while inverting the following $(N-1) \times(N-1)$ matrix: 
<smiles>[B]I([B])[I-]I</smiles>

we find* directly that $\left(\mathrm{B}^{-1}\right)_{j k}=k[1-(j / N)]$ for $j \geqq k$ and $j[1-(k / N)]$ for $j \leqq k$. On the other hand, $\left(\mathrm{B}^{-1}\right)_{i k}$ can also be calculated by first finding the eigenvectors and eigenvalues of $B$. Equating these two results we establish (C7).

(C2) folow ws from (C1) trivially, (C3) is obtained by adding $(\mathrm{C} 1)$ and $(\mathrm{C} 2)$. (C4) is verified by letting $N=2 M$ in (C3). (C5) can be proven by first showing that $I(2 K) \equiv K^{2}$ $+I(K)$ for all positive integer values of $K$, and then finding the solution. To show the above functional equation, let $N$ $=2 \mathrm{~K}$ in $(\mathrm{C} 5)$, separate odd and even summations, and use (C1) with $s=K$ to obtain the sum over odd terms. The proof of (C6) is based on (C4)

\section{NOTE ADDED IN PROOF}

$\mathscr{P}(q, t)$ decays exponentially in the small- $q$ region with a decay constant $q^{2} D$ where $D$ is the translational diffusion coefficient. In light scattering in this $q$-region, the experimental time interval is adjusted such that $q^{2} D t$ ranges approximately from $0.05-0.1$ to $4-5$, and $\mathscr{S}(q, t)$ decays appreciably but yet still above the noise level. Hence, we let $q \rightarrow 0$ and $t \rightarrow \infty$ keeping $q^{2} t$ fixed (Morkov Limit ${ }^{16}$ ) in the Langevin equation (14) in order to calculate the diffusion coefficient. The result is

* This form of $\mathrm{B}^{-1}$ was pointed out to us by Professors E Ozizmir and K. Imre at Richmond College, Staten Island, NY, USA.
$D=D_{0}-D_{1}$ where

$$
D_{0}=\lim _{q \rightarrow 0} \Omega(q) / q^{2}
$$

and

$$
D_{1}=\lim _{q \rightarrow 0} \frac{1}{q^{2}} \int_{0}^{\infty} \mathrm{d} u \varphi(q, u)
$$

It is shown ${ }^{10,42}$ that $D_{0}$ corresponds to Kirkwood's approximation ${ }^{14}$ of the diffusion coefficient. Fixman ${ }^{52}$ has pointed out to us that $D_{0}$, in as much as it is obtained from the initial slope $\Omega(q)$, corresponds to the short-time diffusion coefficient that does not take into account the coupling between the internal and centre of mass motions of the polymer molecule. The correction $D_{1} / D_{0}$ arising from this coupling has been estimated ${ }^{52,53}$ not to be more than $1.4 \%$ for flexible chains. In the text of this paper, no distinction is made between $D_{0}$ and $D_{1}$. The difference between $D_{0}$ and $D_{1}$ leads to a correction in the extraction of $\Omega(q)$ from $\mathscr{S}(q, t)$ data through a known shape function, $f(q, \tau)$. The latter was calculated in the text using the exact expression of $\mathscr{S}(q, t)$ for an unperturbed closed chain with preaveraged Oseen tensor. Since $D_{1}$ vanishes exactly in this case (to be shown elsewhere), $f_{c}(q, \tau) \rightarrow-\tau$ as $q R_{g} \rightarrow 0$ (see Figure 4). The correct shape function for an open chain, which is not available analytically as a function of $q$ at present, should approach $f_{0}(q, \tau) \rightarrow-\left(D / D_{0}\right) \tau$ as $q R_{g} \rightarrow 0$. Since $f_{c}(q, \tau)$ and $f_{0}(q, \tau)$ become identical in the intermediate and large $q$-regions, as demonstrated in the text, no correction is needed in extracting $\Omega(q)$ from $\mathscr{S}(q, t)$ data in these $q$-regions. 